\title{
On the Accurate Large-scale Simulation of Ferrofluids
}

\author{
LIBO HUANG, TORSTEN HÄDRICH, DOMINIK L. MICHELS, KAUST
}
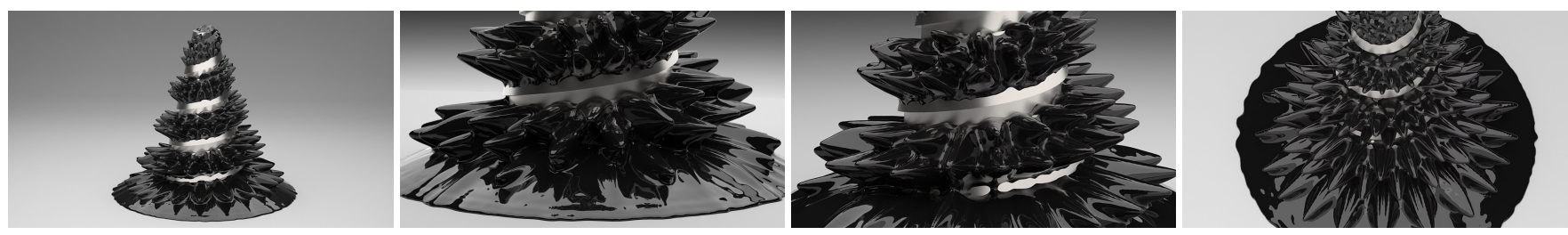

Fig. 1. Our approach can accurately reproduce the observation that real ferrofluid is literally climbing up a steel helix placed above a strong electromagnet. This figure shows the final results of our simulation of this scenario rendered from different viewpoints.

We present an approach to the accurate and efficient large-scale simulation of the complex dynamics of ferrofluids based on physical principles. Ferrofluids are liquids containing magnetic particles that react to an external magnetic field without solidifying. In this contribution, we employ smooth magnets to simulate ferrofluids in contrast to previous methods based on the finite element method or point magnets. We solve the magnetization using the analytical solution of the smooth magnets' field, and derive the bounded magnetic force formulas addressing particle penetration. We integrate the magnetic field and force evaluations into the fast multipole method allowing for efficient large-scale simulations of ferrofluids. The presented simulations are well reproducible since our approach can be easily incorporated into a framework implementing a Fast Multipole Method and a Smoothed Particle Hydrodynamics fluid solver with surface tension. We provide a detailed analysis of our approach and validate our results against real wet lab experiments. This work can potentially open the door for a deeper understanding of ferrofluids and for the identification of new areas of applications of these materials

CCS Concepts: • Computing methodologies $\rightarrow$ Physical simulation

Additional Key Words and Phrases: Computational Electromagnetics, Fast Multipole Method (FMM), Ferrofluids, Fluid Mechanics, Large-scale Simulations, Magnetic Fluids, Maxwell's equations, Natural Phenomena, NavierStokes Equations, Numerical Simulations, Smoothed Particle Hydrodynamics (SPH), Validation Experiments.

\section{ACM Reference Format:}

Libo Huang, Torsten Hädrich, Dominik L. Michels. 2019. On the Accurate Large-scale Simulation of Ferrofluids. ACM Trans. Graph. 38, 4, Article 93 (July 2019), 15 pages. https://doi.org/10.1145/3306346.3322973

\section{INTRODUCTION}

Ferrofluids were invented in the early 1960s by NASA scientists to pump fuel into spacecrafts in low gravity environments without mechanical actions. Today, these fluids are mostly known to the

Author's address: Libo Huang, Torsten Hädrich, Dominik L. Michels, KAUST, Visual Computing Center, Thuwal 23955, KSA.

Permission to make digital or hard copies of part or all of this work for personal or classroom use is granted without fee provided that copies are not made or distributed for profit or commercial advantage and that copies bear this notice and the full citation on the first page. Copyrights for third-party components of this work must be honored. For all other uses, contact the owner/author(s).

(C) 2019 Copyright held by the owner/author(s).

0730-0301/2019/7-ART93

https://doi.org/10.1145/3306346.3322973 public for its peculiar behavior when interacting with magnetic fields. Because of the interesting geometric structure, they are widely used in artwork (as, e.g., in the SIGGRAPH Art Gallery [Kodama 2008]), science exhibitions, and in several advertisements. Due to the complexity of the movements of their spikes, it becomes impossible for an artist to create a lively animation by intuition. A fast and accurate ferrofluid simulator can not only be used for animation, but also to explore, and perhaps better understand, the interplay between the magnetic field, the geometry of the external object, and the resulting surface shape/topology of the ferrofluid. However, realistic simulations of the ferrofluid dynamics are not yet available. In this contribution, for the first time, we achieved the level of realism to bring its fascination from reality to the virtual world. With such a simulation tool at hand, we can further design the magnetic field and external objects in the virtual world to create the desired shapes in reality.

Next to their artistic applications, ferrofluids are employed in different scenarios described in the literature [Nochetto et al. 2016a; Raj et al. 1995] ranging from applications in acoustics, instrumentation, lubrication, vacuum technology, vibration damping [Miwa et al. 2003], to radar absorbing materials [Vinoy and Jha 1996]. Several applications are also touching the field of visual computing, for example, ferrofluids are used for magnetic resonance imaging contrast enhancement and the construction of adaptive deformable mirrors [Brousseau et al. 2007]. Additional applications can be found in the field of micro- and nanoelectronics [Hartshorne et al. 2004; Zahn 2001].

The interesting dynamics of ferrofluids are caused by the interplay of an external magnetic field and the surface tension, forming a pattern of characteristic spikes. Figure 2 illustrates the influence of the magnetic field strength and surface tension on the shape of the spikes: a strong surface tension force smooths the contour, while a strong field strength increases the height of the spikes. These effects are further coupled with fluid motion finally leading to a highly complex dynamical behavior. Given this degree of complexity, the large-scale numerical simulation of ferrofluids can potentially open the door for a deeper understanding of ferrofluids and for the identification of new areas of applications of these materials.

In this contribution, we aim for the large-scale simulation of ferrofluid dynamics. The simulation of magnetism-based effects is 
an active subject of research within the visual computing community. In previous work, the authors are mostly focusing on magnetized rigid bodies, e.g., Thomaszewski et al. [2008] simulated magnetic rigid body interactions by subdividing a rigid body into small cells, and magnetize each cell only using external fields to calculate the net force. The recent work of Kim et al. [2018] addresses the dynamic magnetization process of the material improving the quality and stability of such simulations. However, both models may lead to instabilities when particles come close.

Moreover, in the absence of a magnetic field, our simulation of a ferrofluid is reduced to a Smoothed Particle Hydrodynamics (SPH) fluid simulation with surface tension. Such large-scale fluid simulations are studied in detail within the computer graphics community.

Our magnetic force model is different from previous particle based methods in the graphics and physics literature since we use a smooth magnet instead of a point dipole magnet, for each particle. This eliminates the magnetic field singularities at the dipole positions, and accounts for the self-generated magnetic field when considering the magnetization process. Additionally, our magnetic force model can accurately calculate the forces, in particular if two particles overlap. Our forces are bounded in contrast to point magnet forces. Such property allows for the natural extension to other magnetizable continuum such as magnetic slime and paste by changing the fluid solver to a viscous material solver.

Our specific technical contributions are as follows. We devise a novel approach to robustly calculate the magnetic field generated by multiple magnetizable bodies exposed to an external magnetic field. Moreover, we present a magnetic force model between magnetized particles that enables the accurate formation of the ferrofluids' spikes. Finally, we integrate the Fast Multipole Method (FMM) into the magnetic field and force calculations enabling efficient and accurate large-scale simulations of ferrofluids. Our simulations are well reproducible since our approach can be easily incorporated into a framework implementing a FMM and a SPH fluid solver with surface tension. To further ensure reproducibility, we disclose all relevant details of our implementation and provide all physical and numerical parameters. Moreover, we present a detailed derivation of our method in the supplemental material. We provide a detailed analysis of our approach and validate the results of our simulations against data obtained in real experiments carried out in the wet lab.

\section{RELATED WORK}

As in the case of regular, i.e., non-magnetizable fluids, the dynamics of ferrofluids and its numerical simulation have been studied in different scientific communities including applied mathematics, computational physics (in particular, in computational fluid dynamics (CFD)), as well as in visual computing.

\subsection{Ferrofluids Simulation}

Within the scope of visual computing, Ishikawa et al. [2013] employed an SPH approach to simulate the fluid's motion and treated each particle as a perfect magnetic dipole. After calculating the magnetic moment for each particle, a procedural method is applied to elevate the surface according to the magnetic strength to generate the spikes. Ishikawa et al. [2012] seeds the fluid's surface with

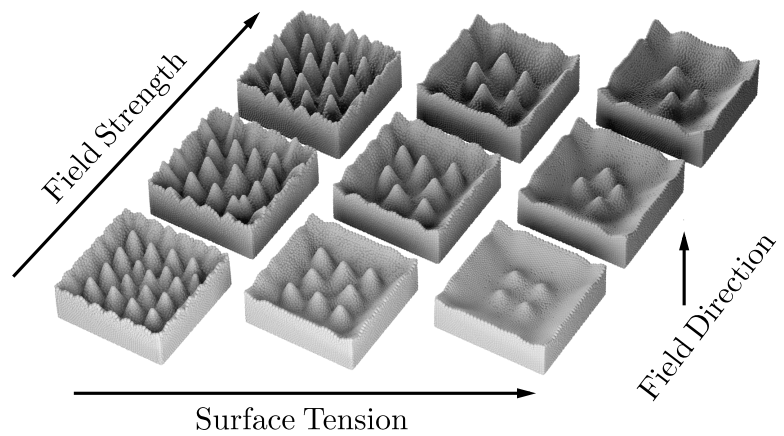

Fig. 2. If a magnetic field is applied, the ferrofluid forms its characteristic spikes. This is illustrated here for different surface tension and magnetic field strengths using a vertical homogeneous magnetic field. A strong surface tension force smooths the contour while a strong magnetic field strength increases the height of the spikes.

particles and generate dynamical spikes by moving the particles according to the strength of the magnetic field. Both methods are not based on self-evident physical laws leading to a limited physical accuracy. This results in significant shortcomings including an unnatural look of the final shapes of the spikes.

Within the computational physics communities, Yoshikawa et al. [2010] employed the moving particle semi-implicit (MPS) method for incompressible free surface flows and reused the particles as nodes in a finite element method (FEM) solver for computing the electromagnetic interactions. They were able to produce only one spike purely based on physical principles without additional geometric modeling. However, the ever-changing mesh topology impedes an efficient simulation. Mitsufuji et al. [2016] also achieved the formation of a single bulge rather than multiple spikes using a particle method for simulating the fluid's dynamics. They formulated the magnetization problem as a system of linear equations as done in our work presented in this contribution. There are additional meshbased examples [Cao and Ding 2014; Gollwitzer et al. 2007; Lavrova et al. 2006, 2008] successfully generating multiple spikes in the presence of an external field. They alternately solve the magnetization of the fluid given the surface profile and use the magnetic field to update the deformation of the surface profile. However, their formation process of spikes is an evolving sequence towards the equilibrium shape rather than a dynamic system advancing in time. Such mesh-based methods also suffer from topology changes in dynamical simulations.

Within the applied mathematics disciplines, Nochetto et al. [2016a] proposed a diffuse interface model for two-phase ferrofluid flows which is representative for the usual approach taken in this field: their solution is given as a function of time rather than iterating towards equilibrium shapes as done in most of the work presented in the computational physics communities. The authors provide a comprehensive analysis on the stability of their scheme and the convergence of the solution. Their model is sufficient to produce many spikes in a two dimensional example without making any nonphysical assumption about the geometric structure of the spikes. A 
FEM approach is used in order to solve their set of partial differential equations. Nochetto et al. [2016b] simulate the ferrofluid using Rosensweig's equation [1997]. However, it is only applied to one phase, and as a consequence no spikes can be formed.

\subsection{Fluid Solvers}

Our approach for the simulation of ferrofluids requires the utilization of a particle-based fluid solver. For this, we use the SPH solver described by Adami et al. [2012]. The authors provide a versatile way to handle complex boundaries easily. Its accuracy and efficiency is sufficient to prove that our ferrofluid force model is qualitatively accurate. In essence, it is still a weakly-compressible $\mathrm{SPH}$ (WCSPH) solver. We would like to mention, that there are more efficient SPH solvers available, e.g., DFSPH [Bender and Koschier 2017], IISPH [Ihmsen et al. 2014a], and PCISPH [Solenthaler and Pajarola 2009]. Some other aspects of SPH methods can also be improved, e.g., viscosity forces [Peer et al. 2015; Peer and Teschner 2017], boundary handling [Akinci et al. 2012; Band et al. 2018a,b], and data structures [Ihmsen et al. 2011]. Recent advances in CFD include particle shifting [Lind et al. 2012; Sun et al. 2017] and density diffusion [Antuono et al. 2012]. The possible improvement list is far from being exhaustive, and we refer the reader to surveys in computer graphics [Ihmsen et al. 2014b; Koschier et al. 2019], in mesh-free methods [Belytschko et al. 1996], and in CFD [Monaghan 1992, 2012] for further information.

We believe in principle that next to SPH, particle-based fluid solvers handling incompressibility should also work with our approach; see, e.g., [Fu et al. 2017; Hu et al. 2018; Jiang et al. 2017; Zheng et al. 2015; Zhu and Bridson 2005].

To ensure the formation of the accurate shapes of the spikes, surface tension has to be incorporated into the simulation. In this regard, a few surface tension models are available: Müller et al. [2003] incorporated surface tension in their SPH framework using the Laplacian of a so-called color field, which is equal to one inside the fluid and zero outside. He et al. [2014] used the surface energy to calculate the surface tension based on the gradient of the color field. These methods are making use of the geometry of the liquid. In contrast, other authors [Akinci et al. 2013; Tartakovsky and Meakin 2005; Yang et al. 2017] employ a pair-wise force between particles, handling the surface tension in a microscopic way: SPH particles attract and repel each other depending on their relative distances, and particles on the surface are exposed to imbalanced forces towards the inside, which become the surface tension forces In this contribution, we incorporate the surface tension model as described by Yang et al. [2017] which is based on pair-wise forces with additional anisotropic filtering for poor-sampled regions.

\section{FERROFLUID DYNAMICS}

The formation of spikes in ferrofluids can be explained by two facts. First, magnetization: magnetizable objects concentrate magnetic field lines around them. Second, magnetic forces: a small magnet tends to be aligned with the magnetic flux density, and is attracted to regions with denser field lines, for example to the center region as illustrated in Figure 3.

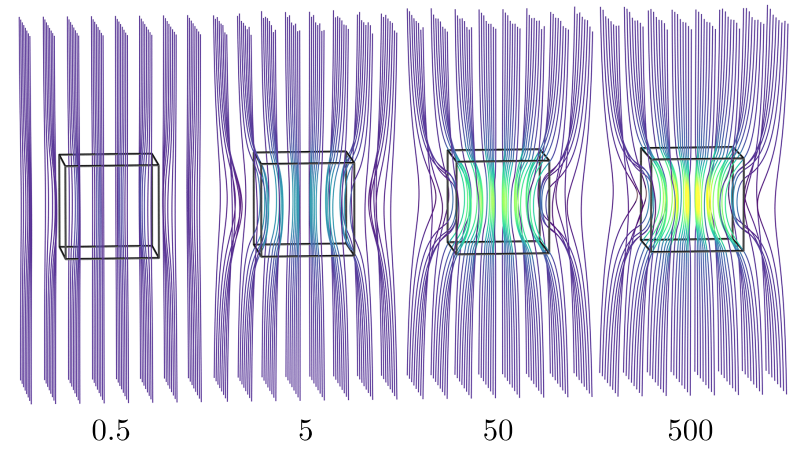

Fig. 3. Illustration of the field lines of a constant vertical magnetic field under the influence of cubes with different susceptibility $\chi$. The susceptibility set to $\chi=0.5,5,50,500$ (from left to right). Typical ferrofluids have a susceptibility between 1 and 5 [Rosensweig 1987]. The field lines are generated by advancing tracing particles along the magnetic flux density direction $\boldsymbol{B}$. Please note that four independent fields are plotted.

A ferrofluid is a deformable and paramagnetic continuum. It is non-magnetic in the absence of an external magnetic field since the total magnetic field caused by the randomly moving magnetic nanoparticles is canceled out. If an external field is present, these magnetic nanoparticles are still moving randomly but rotate statistically to a dominant direction within a short relaxation time, and the fluid becomes magnetized concentrating the field lines. Whenever there is a small bump on the ferrofluid surface caused by a minor perturbation, it bends the field lines and attracts the molecules in its neighborhood forming a larger bump, eventually becoming a spike. The growth of such a spike is limited by gravity, external magnetic forces, and surface tension.

Our approach for the simulation of ferrofluids consists of two components: a fluid solver incorporating surface tension and a magnetic force solver. We discretize the fluid as overlapping smooth magnetizable particles. The fluid solver with surface tension and magnetic forces evolves the motion of these particles .

\subsection{Magnetization}

We derive the magnetization equation from Maxwell's equations, then use the smooth magnets concept to discretize and solve the equation. We use a bold letter for a vector (tensor), and its thin version with superscripts for its components in its canonical coordinates. We add a tilde symbol to denote the same vector (tensor) in other coordinates $(\tilde{\boldsymbol{m}}, \boldsymbol{m})$. We use the subscript to identify the associated index of a particle.

The relaxation time is much smaller than our numerical integration step sizes, so that the magnetization process achieves the equilibrium instantly. The strength of a magnetized object is described by the magnetization field $\boldsymbol{M}=\mathrm{d} \boldsymbol{m} / \mathrm{d} V$, which is the density of the magnetic moment $\boldsymbol{m}$ (one can naively equate $\boldsymbol{m}$ to tiny magnets). Here we use a simplified model and assume a linear relationship between the magnetization of the ferrofluid and the magnetic field:

$$
\boldsymbol{M}=\chi(\boldsymbol{r})\left(\boldsymbol{H}_{\mathrm{ext}}+\boldsymbol{H}_{\mathrm{ferro}}(\boldsymbol{M})\right),
$$




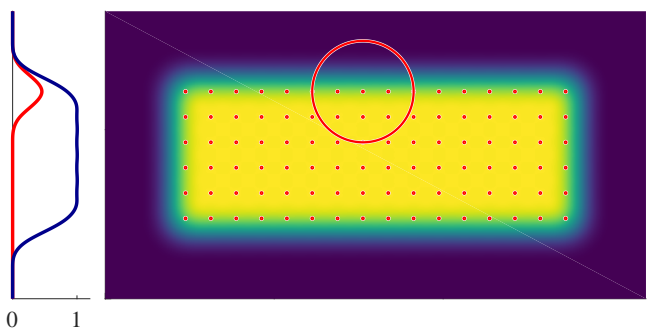

Fig. 4. Illustration of the normalized magnetic susceptibility $\chi(\boldsymbol{r})$. The distance between adjacent particles is given by $h$, the kernel size parameter (see Eq. (10)). The circle indicates the support of the kernel function. On the left side, the cross-section in the middle is plotted as a blue curve along with a kernel function $W$ in Eq. (10) plotted in red. The susceptibility is constant within the center region of the continuum and drops quickly down to zero beyond the boundary. A transition layer (the sloped region in the left blue curve) of approximately $2 h$ is observed.

where $\boldsymbol{H}_{\text {ext }}$ is the external magnetic field, $\boldsymbol{H}_{\text {ferro }}$ is the magnetic field generated by the magnetized ferrofluid, and $\chi$ is the volume magnetic susceptibility field which is zero outside the fluid and constant inside the fluid. We emphasize here, that $\chi: r \mapsto \chi(r)$ is a function mapping the spatial position $r$ onto the corresponding susceptibility. Later, $\chi$ can be a constant.

The magnetization problem asks for $\boldsymbol{M}$ given a fixed external magnetic field $\boldsymbol{H}_{\text {ext }}$. From Eq. (1), it is clear that the magnetization $\boldsymbol{M}$ is a function of $\boldsymbol{H}_{\text {ferro }}$ which is generated by the ferrofluid itself. We approach this complicated phenomenon by starting from a simpler question asking for the field $\boldsymbol{H}_{\text {ferro }}$ generated by the ferrofluid given a known magnetization $\boldsymbol{M}$. The related Maxwell's equations are

$$
\begin{aligned}
\nabla \cdot \boldsymbol{B} & =0, \\
\boldsymbol{B} & =\mu_{0}(\boldsymbol{H}+\boldsymbol{M}), \\
\nabla \times \boldsymbol{H} & =\boldsymbol{J}_{f}+\frac{\partial \boldsymbol{D}}{\partial t},
\end{aligned}
$$

where $B$ is the magnetic flux density, $\mu_{0}=4 \pi \cdot 10^{-7} \mathrm{NA}^{-2}$ is the permeability of the vacuum, $\boldsymbol{H}=\boldsymbol{H}_{\text {ext }}+\boldsymbol{H}_{\text {ferro }}$ is the total magnetic field, $\boldsymbol{J}_{f}$ is the free current density, $\boldsymbol{D}$ is the electric displacement field, and $t$ is the time parameter. The moving ferrofluid's magnetic field is too weak to cause significant eddy currents and magnetic damping. Therefore we neglect the associated time derivative part. On the other hand, the ferrofluid is non-conductive, so the free current density is only associated with external magnetic sources. After these simplifications,

$$
\begin{aligned}
\nabla \cdot\left(\boldsymbol{M}+\boldsymbol{H}_{\mathrm{ext}}+\boldsymbol{H}_{\text {ferro }}\right) & =0, \\
\nabla \times\left(\boldsymbol{H}_{\mathrm{ext}}+\boldsymbol{H}_{\text {ferro }}\right) & =\boldsymbol{J}_{f} .
\end{aligned}
$$

The external field solely generated by the flow of electric charges is unaffected if we remove the field caused by the ferrofluid. Therefore, another set of equations for the external field emerges:

$$
\begin{aligned}
\nabla \cdot H_{\text {ext }} & =0, \\
\nabla \times H_{\text {ext }} & =J_{f} .
\end{aligned}
$$

We substitute Eq. (5-6) into Eq. (3-4)

$$
\begin{aligned}
\nabla \cdot \boldsymbol{H}_{\text {ferro }} & =-\nabla \cdot \boldsymbol{M}, \\
\nabla \times \boldsymbol{H}_{\text {ferro }} & =0 .
\end{aligned}
$$

The last equation indicates that $\boldsymbol{H}_{\text {ferro }}$ is curl-free, so it can be represented by the negative gradient of another scalar function $\phi$, i.e., $\boldsymbol{H}_{\text {ferro }}=-\nabla \phi$.

We turn this problem into Poisson's equation:

$$
\begin{aligned}
\nabla^{2} \phi(\boldsymbol{r}) & =\nabla \cdot \boldsymbol{M}, \\
\left.\phi\right|_{\infty} & =0 .
\end{aligned}
$$

Here, we apply zero Dirichlet boundary conditions at infinity because of the huge distance from the sources. Such quasi-static assumptions leading to a scalar Poisson's equation were also used in previous physics literature [Cao and Ding 2014; Gollwitzer et al. 2007; Odenbach 2008]. In the next step, we discretize Eq. (7).

In our model, the magnetic continuum is represented by thousands of small particles. Each particle represents a density distribution (cloud) of billions of magnetic nanoparticles sharing the same direction. From now on, a "particle" represents a cloud of magnetic nanoparticles. Each particle has the same spherically symmetric Gaussian-like density distribution function $W$ known as the socalled "kernel"; see Figure 4, left. The positions of these particles are also used in SPH fluid simulations in which the uniform spacial distribution of particles is guaranteed. Hence, the magnetic susceptibility $\chi$, which is proportional to the density of the magnetic nanoparticles, is constant inside the fluid and drops rapidly down to zero outside the region occupied by the fluid; see Figure 4.

The magnetization $\boldsymbol{M}$ in Eq. (7) is discretized. Assume we have $N$ particles, and each particle enumerated with an index $i$ is magnetized according to the magnetic field at its center $\boldsymbol{r}_{i}$ :

$$
\begin{aligned}
\boldsymbol{M}(\boldsymbol{r}) & =\sum_{i=1}^{N} \boldsymbol{m}_{i} W\left(\boldsymbol{r}-\boldsymbol{r}_{i}, h\right), \\
\boldsymbol{m}_{i} & =V \chi\left(\boldsymbol{H}_{\mathrm{ext}}\left(\boldsymbol{r}_{i}\right)+\boldsymbol{H}_{\mathrm{ferro}}\left(\boldsymbol{r}_{i}\right)\right),
\end{aligned}
$$

where $\boldsymbol{m}_{i}$ is the total magnetic moment of the particle enumerated with the index $i, V=(\Delta x)^{3}$ is the volume of each particle as all particles are initially placed on a grid with $\Delta x$-spacing, $\chi$ is not a field but a constant for this liquid now, and $W\left(\boldsymbol{r}-\boldsymbol{r}_{i}, h\right)$ is the Gaussian-like kernel function centered around $\boldsymbol{r}_{i}$. The parameter $h$ controls the size of the kernel. We make use of the kernel function [Monaghan 1992]

$$
W(\boldsymbol{r}, h)=\frac{1}{h^{3}} w\left(\frac{r}{h}\right), \quad \int W(\boldsymbol{r}, h) \mathrm{d} \boldsymbol{r}=1
$$

with $r:=|\boldsymbol{r}|$ and $w(q): \mathbb{R} \ni q \mapsto w(q) \in \mathbb{R}$,

$$
w(q)=\frac{1}{\pi} \begin{cases}0.25(2-q)^{3}-(1-q)^{3} & 0 \leq q<1, \\ 0.25(2-q)^{3} & 1 \leq q<2, \\ 0 & q \geq 2 .\end{cases}
$$

After substituting Eq. (8) into Eq. (7) we obtain

$$
\nabla^{2} \phi(\boldsymbol{r})=\nabla \cdot \sum_{i=1}^{N} \boldsymbol{m}_{i} W\left(\boldsymbol{r}-\boldsymbol{r}_{i}, h\right) .
$$

The field generated by the ferrofluid is the superposition of the fields generated by all ferrofluid particles. The details of solving 
Eq. (11) with one particle are in the supplemental material (S1). For a single particle with kernel $W$ placed at the origin with a total magnetic moment $\boldsymbol{m}$, the magnetic field at $\boldsymbol{r}$ is given by

$$
\boldsymbol{H}(\boldsymbol{r}, \boldsymbol{m})=(\hat{\boldsymbol{r}} \cdot \boldsymbol{m})\left(W_{\mathrm{avr}}(r)-W(r)\right) \hat{\boldsymbol{r}}-\frac{W_{\mathrm{avr}}(r)}{3} \boldsymbol{m},
$$

with $\hat{\boldsymbol{r}}:=\boldsymbol{r} / \boldsymbol{r}$ for $r>0$, and by $\boldsymbol{H}(\boldsymbol{r}, \boldsymbol{m})=-W(0) / 3 \boldsymbol{m}$ for $r=0$. Here, we make use of the average density within $r$ :

$$
W_{\text {avr }}(r)=\left(\frac{4 \pi}{3} r^{3}\right)^{-1} \int_{0}^{r} 4 \pi \xi^{2} W(\xi) \mathrm{d} \xi .
$$

$W_{\text {avr }}$ depends on Eq. (10) and can be evaluated similarly to the density function $W$, but $w(r / h)$ in Eq. (10) is replaced by the following expression:

$$
w_{\mathrm{avr}}(q)=\frac{1}{\pi} \begin{cases}\frac{1}{40}\left(15 q^{3}-36 q^{2}+40\right) & 0 \leq q<1, \\ \frac{-3}{4 q^{3}}\left(\frac{q^{6}}{6}-\frac{6 q^{5}}{5}+3 q^{4}-\frac{8 q^{3}}{3}+\frac{1}{15}\right) & 1 \leq q<2, \\ \frac{3}{4 q^{3}} & q \geq 2 .\end{cases}
$$

Using Eq. (12) as the solution for a single particle, we are ready to solve the magnetization of the ferrofluid in an external magnetic field. We formulate this as a least squares problem. Before arriving at the objective function, we first clarify some variables and matrices. The variables of this objective function are the magnetic flux density at each particle position divided by the vacuum permeability, i.e., $\boldsymbol{B}\left(\boldsymbol{r}_{i}\right) / \mu_{0}$. Using Eq. $(2,8,12)$ we obtain

$$
\frac{B\left(\boldsymbol{r}_{i}\right)}{\mu_{0}}=\boldsymbol{H}_{\mathrm{ext}}\left(\boldsymbol{r}_{i}\right)+\sum_{j=1}^{N}\left(\boldsymbol{H}\left(\boldsymbol{r}_{i}-\boldsymbol{r}_{j}, \boldsymbol{m}_{j}\right)+\boldsymbol{m}_{j} W\left(\boldsymbol{r}_{i}-\boldsymbol{r}_{j}, h\right)\right) .
$$

Please note that according to Eq. (12), $\boldsymbol{H}\left(\boldsymbol{r}, \boldsymbol{m}_{i}\right)$ is linear with respect to the magnetic moment $\boldsymbol{m}_{i}$. Hence, Eq. (13) can be written in a matrix form:

$$
b=G m+h_{\mathrm{ext}},
$$

where $\boldsymbol{m}$ and $\boldsymbol{b}$ are vectors with $3 N$ scalar elements, and $G$ is a $3 N \times 3 N$ dense matrix given as a function of all particle positions $\boldsymbol{r}_{i}$. Here, $\boldsymbol{h}_{\text {ext }}$ is also a vector of $3 N$ scalar elements representing $\boldsymbol{H}_{\text {ext }}$ measured at the particle positions.

We express the magnetization using the flux density $\boldsymbol{B}$ instead of the magnetic field $\boldsymbol{H}$ by incorporating Eq. $(1,2,9)$ :

$$
\boldsymbol{m}_{i}=V \frac{\chi}{1+\chi} \frac{\boldsymbol{B}\left(\boldsymbol{r}_{i}\right)}{\mu_{0}}
$$

This can be expressed as a scalar-vector product:

$$
\boldsymbol{m}=\Gamma \boldsymbol{b}, \quad \Gamma=V \frac{\chi}{1+\chi} .
$$

Finally, we substitute Eq. (15) into Eq. (14), and obtain the linear equation system

$$
(G \Gamma-1) b=-h_{\mathrm{ext}},
$$

in which 1 denotes the identity matrix. We are going to solve this equation in the least-squares sense.

\subsection{Force Model}

The needle in a compass receives a torque from the Earth's magnetic field until it points to the Earth's magnetic field direction. A magnetic particle receives the torque in the same way. After we solve the magnetization process, the magnetic moment of a particle is aligned with the magnetic field direction. Therefore, there is no magnetic torque and we can safely only consider the magnetic force. A huge number of magnetic body force models can be found in the literature [Byrne 1977; Engel 2001; Liu 2001; Odenbach 2008; Odenbach and Liu 2001] and previous contributions employing mesh-based methods [Cao and Ding 2014; Gollwitzer et al. 2007; Lavrova et al. 2006, 2008] obtain successful results using these force models. All force models have a surface force term due to the discontinuous body force tensor on the fluid-air interface. Mesh-based methods have a defined boundary and it is easy to attribute this force to the boundary nodes. However, our smooth magnets model has a vague boundary for the liquid (see Figure 4). The surface force term can be calculated as the integral of body forces in the thin boundary ([Byrne 1977], Sec. 2.1). A naive approach to add the theoretical surface force term at the fluid boundary particle center introduces errors because it neglects the major contribution in the smooth region. We accurately calculate this body force integral for a particle analytically for the long range, and numerically when particles overlap. The body forces integral at the boundary particles naturally contains the surface force term. This approach does not need extra effort to detect boundary particles.

The magnetic force on a particle is given by the total magnetic forces received by all its nanoparticles. The Kelvin force (see Table 1 in Byrne's work [1977]) is used for the force density because of its simplicity:

$$
\boldsymbol{F}_{\text {Kelvin }}=\mu_{0} \boldsymbol{M} \cdot \nabla \boldsymbol{H} .
$$

The total force $f_{s \rightarrow t}$ from the source (s) to the target ( $\mathrm{t}$ ) is given by

$$
\begin{aligned}
f_{s \rightarrow t} & =\int \boldsymbol{F}_{\text {Kelvin }} \mathrm{d} \boldsymbol{r}=\mu_{0} \int \boldsymbol{M}_{t}(\boldsymbol{r}) \cdot \nabla \boldsymbol{H}_{s}(\boldsymbol{r}) \mathrm{d} \boldsymbol{r} \\
& =\mu_{0} \boldsymbol{m}_{t} \cdot \int W\left(\boldsymbol{r}-\boldsymbol{r}_{t}, h\right) \nabla \boldsymbol{H}\left(\boldsymbol{r}-\boldsymbol{r}_{s}, \boldsymbol{m}_{s}\right) \mathrm{d} \boldsymbol{r},
\end{aligned}
$$

where the integration only covers regions with $\boldsymbol{M}_{t} \neq 0$. The source and target center locations are given by $\boldsymbol{r}_{s}$ and $\boldsymbol{r}_{t}$. Using Eq. (12), $\nabla \boldsymbol{H} \in \mathbb{R}^{3 \times 3}$ is determined analytically:

$$
\begin{aligned}
\nabla \boldsymbol{H}(\boldsymbol{r}, \boldsymbol{m}) & =\left(\mathbf{1}\left(\boldsymbol{r}^{\top} \boldsymbol{m}\right)+\mathbf{r m}^{\top}+\mathbf{m r}^{\mathrm{T}}\right) A(r)+\boldsymbol{r}\left(\boldsymbol{r}^{\top} \boldsymbol{m}\right) \frac{\boldsymbol{r}^{\top}}{r} A^{\prime}(r), \\
A(r) & =\frac{W_{\mathrm{avr}}(r)-W(r)}{r^{2}}, \\
A(r)^{\prime} & =5 \frac{W(r)}{r^{3}}-5 \frac{W_{\mathrm{avr}}(r)}{r^{3}}-\frac{1}{r^{2}} \frac{\mathrm{d} W(r)}{\mathrm{d} r},
\end{aligned}
$$

where a temporary scalar $A$ is used and $r=|\boldsymbol{r}|$, and $\mathbf{1} \in \mathbb{R}^{3}$ denotes the identity matrix. It can be used to validate $\nabla \cdot(\boldsymbol{H}+\boldsymbol{M})=0$.

For $\left|\boldsymbol{r}_{t}-\boldsymbol{r}_{s}\right|>4 h$, Eq. (17) has a closed-form solution.

$\boldsymbol{H}\left(\boldsymbol{r}-\boldsymbol{r}_{s}, \boldsymbol{m}_{s}\right)$ is harmonic (divergence-free and curl-free) if $\mid \boldsymbol{r}-$ $\boldsymbol{r}_{s} \mid>2 h$ because of $\boldsymbol{M}_{s}=\boldsymbol{m}_{s} W\left(\boldsymbol{r}-\boldsymbol{r}_{s}, h\right)=0$. If $\left|\boldsymbol{r}_{t}-\boldsymbol{r}_{s}\right|>4 h$, $\nabla \boldsymbol{H}$ is harmonic for the whole integral domain $\left(\left|\boldsymbol{r}-\boldsymbol{r}_{t}\right| \leq 2 h\right)$. Since $W\left(\boldsymbol{r}-\boldsymbol{r}_{t}, h\right)$ is spherically symmetric around $\boldsymbol{r}_{t}$, using the mean value property for harmonic functions, the integral in Eq. (17) can 
analytically be determined as

$$
f_{s \rightarrow t}=\mu_{0} \boldsymbol{m}_{t} \cdot \nabla \boldsymbol{H}\left(\boldsymbol{r}_{t}-\boldsymbol{r}_{s}, \boldsymbol{m}_{s}\right), \text { if }\left|\boldsymbol{r}_{t}-\boldsymbol{r}_{s}\right|>4 h .
$$

It happens to be the force of a point dipole $\boldsymbol{m}_{t}$ at particle center $\boldsymbol{r}_{t}$ in the source field $\boldsymbol{H}_{s}$. If Eq. (19) is used, if $\left|\boldsymbol{r}_{t}-\boldsymbol{r}_{s}\right| \leq 4 h$, we denote it as the "center-only force model".

For $\left|\boldsymbol{r}_{t}-\boldsymbol{r}_{s}\right| \leq 4 h, \boldsymbol{H}_{s}$ is in general not harmonic, so that we make use of numerical integration. However, the numerical integration for each pair of particles is expensive. Instead, we first numerically evaluate the integral for a series of particle distances $\left|\boldsymbol{r}_{t}-\boldsymbol{r}_{s}\right|$, kernel sizes $h$, and particle moment directions $\boldsymbol{m}_{s}, \boldsymbol{m}_{t}$ in advance, and fit the measured data as piece-wise polynomials for later efficient evaluation. The details of the full derivations are shown in the supplemental material (S3). We denote this numerical integration of the Kelvin force Eq. (17) using a fitted black-box function as our "fitted force model".

In the first step, the force in local coordinates is calculated to remove directional dependency. The origin is set up in the center of the source particle; see Figure 5 . Let $\hat{\xi}, \hat{\eta}, \hat{\zeta}$ be the unit vectors of the axes and assume the target particle is on the $\zeta$ axis. The rotation matrix is denoted as $R=(\hat{\xi}, \hat{\eta}, \hat{\zeta})$, so that a vector in the world coordinate $\boldsymbol{m}$ can be connected to its local coordinate counterpart $\tilde{\boldsymbol{m}}$ by $\boldsymbol{m}=\boldsymbol{R} \tilde{\boldsymbol{m}}$ and $\tilde{\boldsymbol{m}}=\boldsymbol{R}^{\top} \boldsymbol{m}$. A tilde indicates that it is the local coordinate vector. In this coordinate system, the same physical law given by Eq. (17) holds:

$$
\tilde{f}_{s \rightarrow t}=\mu_{0} \tilde{\boldsymbol{m}}_{t} \cdot \int W\left(\tilde{\boldsymbol{r}}-\left(0,0,\left|\boldsymbol{r}_{t}-\boldsymbol{r}_{s}\right|\right)^{\mathrm{T}}, h\right) \nabla \boldsymbol{H}\left(\tilde{\boldsymbol{r}}, \tilde{\boldsymbol{m}}_{s}\right) \mathrm{d} \tilde{\boldsymbol{r}} .
$$

In the second step, the dependency on $\tilde{\boldsymbol{m}}_{s}$ and $\tilde{\boldsymbol{m}}_{t}$ is removed. The force is bilinear in both the source and target moments, and Eq. (20) states a mapping: $\mathbb{R}^{3} \times \mathbb{R}^{3} \rightarrow \mathbb{R}^{3}$. This relationship can be described by a third-order tensor $\tilde{\Lambda}$ with 27 entries:

$$
\tilde{f}_{s \rightarrow t}^{\alpha}=\sum_{\beta=1}^{3} \sum_{\gamma=1}^{3} \tilde{\Lambda}^{\alpha \beta \gamma}\left(\left|\boldsymbol{r}_{t}-\boldsymbol{r}_{s}\right|, h\right) \tilde{m}_{s}^{\beta} \tilde{m}_{t}^{\gamma}, \quad \alpha \in\{1,2,3\},
$$

where we use $\alpha, \beta, \gamma$ for denoting vector (tensor) components in local coordinates.

In the third step, we use the integral formula Eq. (20) to determine the tensor $\tilde{\Lambda}^{\alpha \beta \gamma}$ numerically. First, we loop the normalized distance $q$ from 0 to 4 . The target particle is then placed on $(0,0, q h)^{\mathrm{T}}$. The source and target moments $\tilde{\boldsymbol{m}}_{s}$ and $\tilde{\boldsymbol{m}}_{t}$ loop over three directions: $(1,0,0)^{\mathrm{T}},(0,1,0)^{\mathrm{T}},(0,0,1)^{\mathrm{T}}$. For each of the nine combination, the force $\tilde{f}_{s \rightarrow t}$ is determined by Eq. (20). After nine measurements, the tensor value $\tilde{\Lambda}^{\alpha \beta \gamma}$ for this distance $q h$ is obtained. The target particle at $\tilde{\boldsymbol{r}}_{t}=(0,0, q h)^{\mathrm{T}}$ is contained in a cube with an edge length of $4 h$; see Figure 5 . The container is divided into $10 \times 10 \times 10$ cells. We use 1000 quadrature points to obtain the integral results. After the measurements for various $q$ and $h$ we discover that when we fix the normalized distance $q$, the tensor is proportional to $h^{-4}$. Most of the 27 entries in $\tilde{\Lambda}^{\alpha \beta \gamma}$ are zeros. Finally, the six of the seven non-zero entries share the same curve $C_{1}: q \mapsto C_{1}(q)$, and one obtains an unique curve $C_{2}: q \mapsto C_{2}(q)$ :

$$
\begin{aligned}
\tilde{\Lambda}^{311}, \tilde{\Lambda}^{322}, \tilde{\Lambda}^{113}, \tilde{\Lambda}^{223}, \tilde{\Lambda}^{131}, \tilde{\Lambda}^{232} & =C_{1}(q) h^{-4}, \\
\tilde{\Lambda}^{333} & =C_{2}(q) h^{-4} .
\end{aligned}
$$
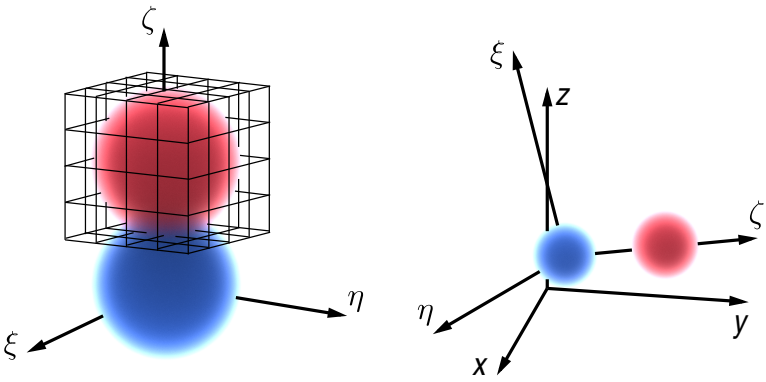

Fig. 5. Illustration of measuring the force from the source kernel (blue) to the target kernel (red) in the rotated coordinates $(\xi, \eta, \zeta)$ (left) and rotated coordinates $(\xi, \eta, \zeta)$ embedded in world coordinates $(x, y, z)$ (right).

The center force Eq. (19) is inaccurate in the near-field $\left|\boldsymbol{r}_{t}-\boldsymbol{r}_{s}\right| \leq$ $4 h$. The difference is illustrated in Figure 6 . The center-force curve is obtained by using the center-force formula Eq. (19) to fit the tensor $\tilde{\Lambda}$ in Eq. (21) in the near-field. The polynomial coefficients are listed in Table 3 and 4 in the appendix.

In the forth step, the summation variables are changed from forces to force tensors. In the large-scale summation, we use the FMM to accelerate the computations. The FMM can only use the positions of the target particles, but summing the forces requires target moments. Therefore we must change the force to a quantity independent of the target magnetic moment $\boldsymbol{m}_{t}$. In order to do so, the summation in Eq. (21) is divided into two parts by introducing a $3 \times 3$ source force tensor $\tilde{T}_{s}$ :

$$
\begin{aligned}
\tilde{f}_{s \rightarrow t}^{\alpha} & =\sum_{\gamma=1}^{3} \tilde{T}_{s}^{\alpha \gamma} \tilde{m}_{t}^{\gamma}, \\
\tilde{T}_{s}^{\alpha \gamma} & =\sum_{\beta=1}^{3} \tilde{\Lambda}^{\alpha \beta \gamma}\left(\left|\boldsymbol{r}_{t}-\boldsymbol{r}_{s}\right|, h\right) \tilde{m}_{s}^{\beta} .
\end{aligned}
$$

Since $\tilde{\Lambda}$ is sparse, one can directly write the force tensor $\tilde{T}_{s}$ in local coordinates:

$$
\tilde{T}_{s}=h^{-4}\left(\begin{array}{ccc}
\tilde{m}_{s}^{3} C_{1}(q) & 0 & \tilde{m}_{s}^{1} C_{1}(q) \\
0 & \tilde{m}_{s}^{3} C_{1}(q) & \tilde{m}_{s}^{2} C_{1}(q) \\
\tilde{m}_{s}^{1} C_{1}(q) & \tilde{m}_{s}^{2} C_{1}(q) & \tilde{m}_{s}^{3} C_{2}(q)
\end{array}\right),
$$

where the superscripts denote the components. We transform the force tensor $\tilde{T}_{s}$ to the world coordinates:

$$
T_{s}=R \tilde{T}_{s} R^{\top},
$$

where $T_{s}$ is the force tensor in world coordinates. $T_{s}$ only depends on the target position, so that it is suitable for the summation to use the fast multipole method:

$$
f_{t}=\left(\sum_{s=1}^{N} T_{s}\left(\boldsymbol{r}_{t}\right)\right) \boldsymbol{m}_{t}
$$

\section{IMPLEMENTATION}

In this section, we provide an overview of our ferrofluid solver and discuss each component in detail. We provide pseudocode for both, the SPH part and the magnetic force computation, to ensure reproducibility. 

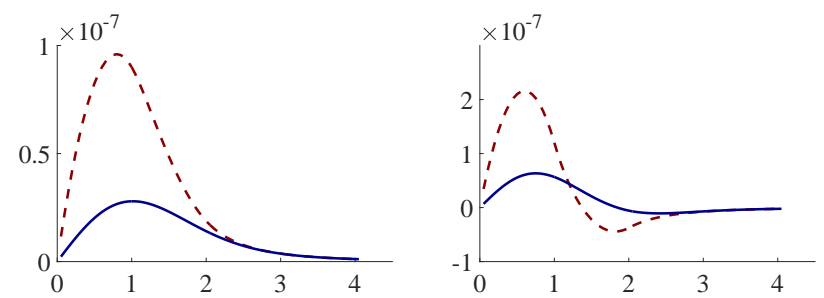

Fig. 6. Illustration of the curves $C_{1}: q \mapsto C_{1}(q)$ (left) and $C_{2}: q \mapsto C_{2}(q)$ (right); see Eq. $(22,23)$. The polynomial coefficients are listed in Table 3 and 4 in the appendix. The red dashed lines represent the center forces while the blue lines represent the fitted forces.

\subsection{Algorithm Overview}

The magnetic force is determined by the particle positions and can directly be added to the particle force in the SPH simulator. We summarize the steps of the fluid solver in Algorithm 1 and the magnetic force solver in Algorithm 2.

In Algorithm 1, the SPH algorithm similar to the work of Adami et al. [2012] is presented: $t$ is the time variable, $t_{\max }$ is the simulation duration, and $N$ denotes the number of particles. The subscript $i$ denotes the index of the associated particle, $\boldsymbol{a}$ is the acceleration, $m$ is the particle mass, and $f$ is the force from a pure fluid simulation. The function MagneticForce is implemented in Algorithm 2. It receives all particles positions, and returns the magnetic force on each particle: $\boldsymbol{v}$ is the velocity, $\boldsymbol{r}$ is the position of each particle, and $\rho$ is the density. It is evolved using the continuity equation eliminating the particle deficiency problem near the surface.

In Algorithm 2, we describe how to obtain the magnetic forces $f$. The input of the algorithm are the positions of the particles $r$, the magnetic kernel size $h$, a constant $\Gamma=V \chi /(1+\chi)$ determined by the particle volume $V$ and the material susceptibility $\chi$. Initially, we place particles on Cartesian grids with spacing $\Delta x$. The volume is obtained by $V=(\Delta x)^{3}$.

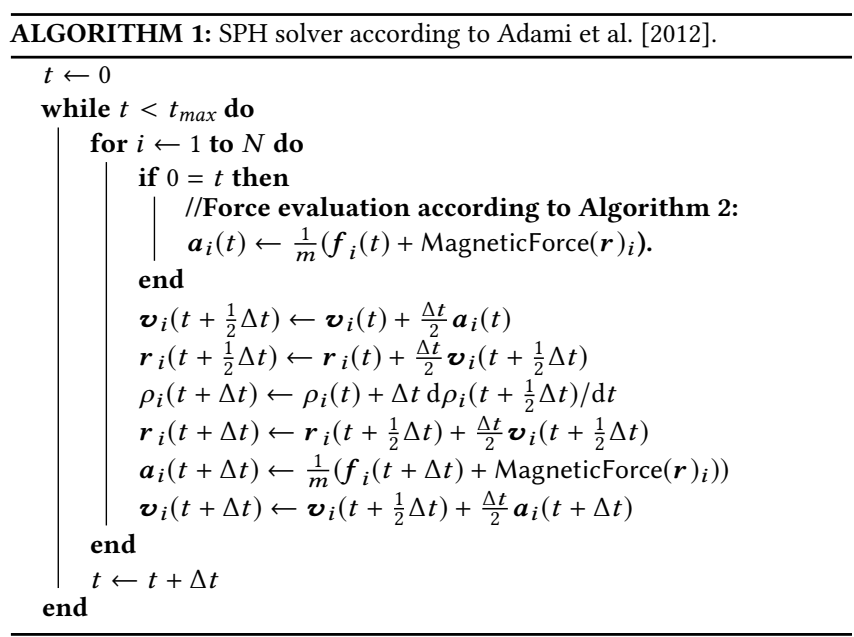

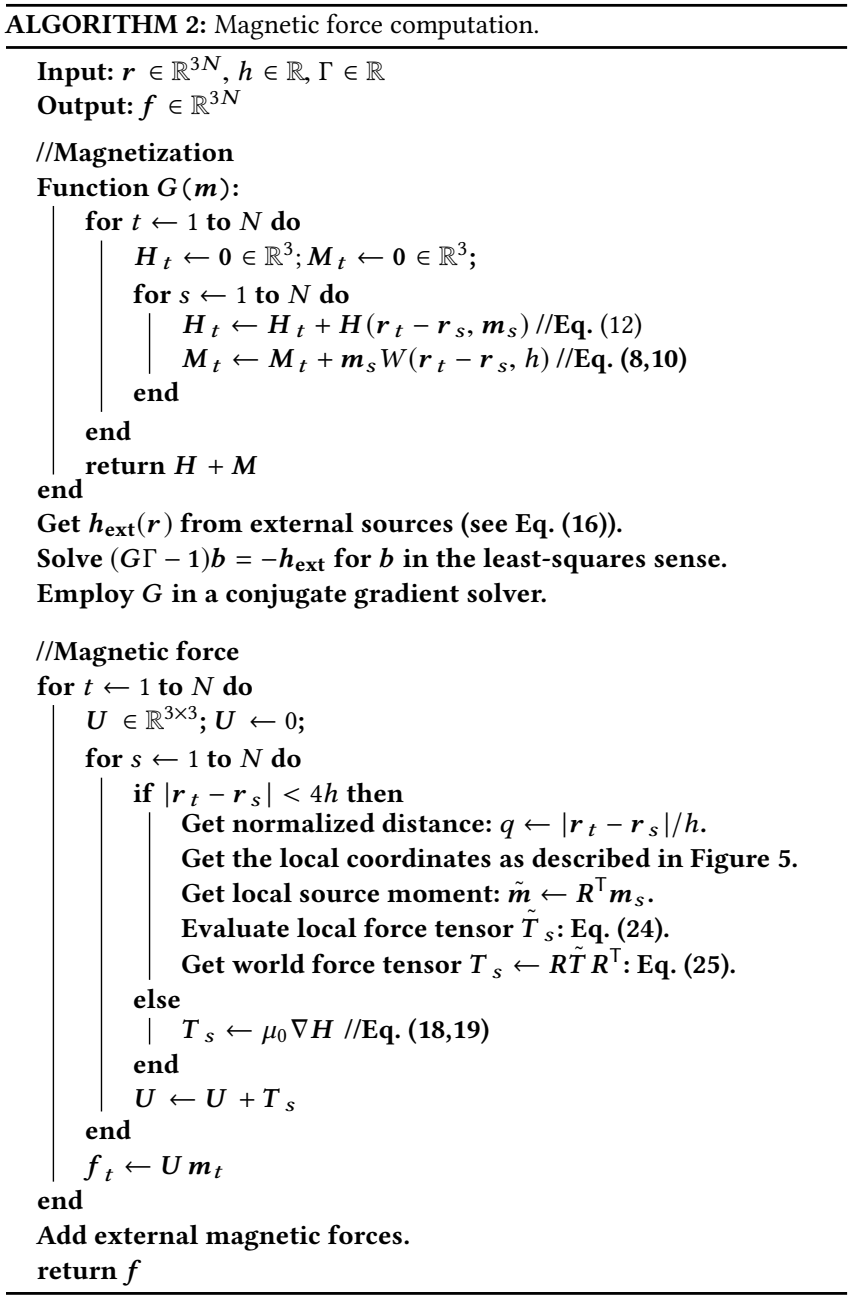

\subsection{Fluid Solver}

The magnetic force solver only relies on particle positions as input. In principle, any Lagrangian fluid solver can be integrated with our magnetic force model. To prove the concept of our ferrofluid model, we use the SPH algorithm as employed by Adami et al. [2012] since it is easy to use because of its simplicity. In this subsection, we first present the details of our SPH solver and then discuss potential improvements.

For the free-surface problem, we do not use the background pressure, and employ an exponent $\gamma=7$ in the pressure state equation. Adami et al. [2012] extrapolate the pressure from the fluid to the wall particles using the gravity as the only non-pressure acceleration. In our cases, magnetic forces and surface tension also contribute to the acceleration. Initially, we added surface tension and magnetic forces next to gravity forces. Then we found that regardless of what part of acceleration we include, as long as it is extrapolated using the fluid pressure according to the authors' approach, the wall pressure causes random ghost force artifacts, pushing particles near the boundary inward. We employ a practical workaround and set the pressure within the wall particles to zero, but still let wall particles 
contribute to the density evolution of fluid particles. Please note that using an alternative SPH method and boundary handling might circumvent this problem. In addition, we note that some advanced SPH solvers [Bender and Koschier 2017; Ihmsen et al. 2014a] avoid excessive attractive pressure forces by clamping the negative pressure. We discuss the pressure forces here as we use it to resolve the expansion artifacts caused by the Kelvin force model. The Kelvin model we used (see Table 1 in Byrne's work [1977]) has a surface force term pointing outward of the ferrofluid. In our first SPH implementation (see Figure 10a), we use weighted mass summations to calculate the density, then use an equation of state (EOS) to get the pressure. We avoid surface artifacts by removing the negative pressure. This implementations penalizes fluid compression but not volume expansion. When we add the Kelvin magnetic force, the surface particles are levitated, causing expansion of fluid volume. Such an expansion is not penalized. Although the surface tension forces point inside the fluid, they are not sufficient to reduce this artifacts. We then changed the SPH implementation to use the continuity equation to evolve the density, and an EOS for pressure (see Figure 10a). Please note that the negative pressure is not removed. This implementation penalizes both compression and expansion of the fluid. Therefore, the levitation artifacts due to the Kelvin magnetic forces are penalized by the continuity condition.

We first set up all particles on uniform Cartesian grid nodes. Then we mark the boundary particles and fluid particles, and finally remove all particles in the air. We choose the quintic spline kernel support radius to be $3 h$, where $h$ is the particle spacing in uniform grids. For the surface tension, we use $k=1$ in the surface tension pair-wise curve in Eq. (6) of Yang et al. [2017]. The whole computational domain is divided into small cells whose edge lengths are $3 h$. The particles in one cell only interact with the neighboring 27 cells.

At the initial phase and whenever the particles move, we find the cell number that a particle reside and record it in each particle. The particles are then sorted according to these cell numbers. Finally, each cell records the begin and end of the particle index after sorting. In our CUDA program, we assign each cell to a block of threads. The particle quantities in the neighboring 27 cells are loaded to the shared memory and are used for the summation by the current cell.

The magnetic force part is much more time consuming than WC$\mathrm{SPH}$. Therefore we treat the WCSPH as a multi-step integrator to advect the particle, and only update the magnetic force every few iterations. We gradually take more (ten) WCSPH steps between magnetic force steps until the results become incorrect. The boundary handling relies on pressure forces which depends on the artificial sound speed. A strong magnetic force requires a larger artificial sound speed to prevent particles from penetrating the boundaries. This leads to a highly stiff system and the sound speed has to be tuned carefully. By using more efficient SPH fluid solvers (PCISPH [Solenthaler and Pajarola 2009], IISPH [Ihmsen et al. 2014a], DFSPH [Bender and Koschier 2017]), a larger time step size can be chosen and sound speed tuning can be avoided. We encourage the reader to try out the possibilities mentioned in the related work section about the recent progress regarding more efficient fluid solvers as well as more accurate boundary handling. In particular, existing incompressible solvers are much faster than WCSPH, enabling the

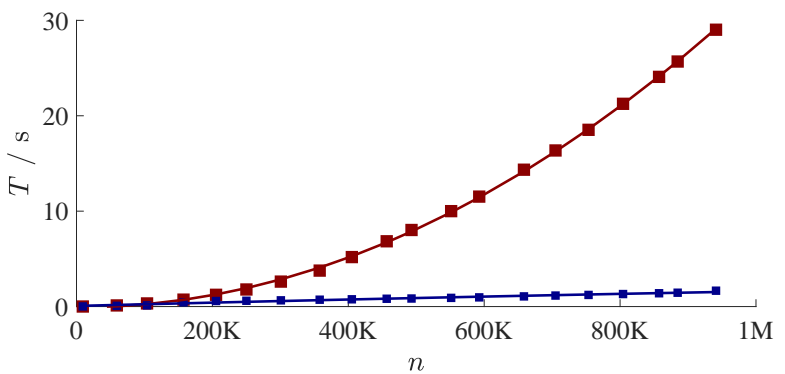

Fig. 7. Runtime comparison between the naive summation (red, $O\left(n^{2}\right)$ ) and the FMM implementation (blue, $O(n)$ ) for one evaluation of $G$ measured for different numbers of particles $n$. T is the runtime in seconds, and $n$ is the number of particles. The curves are fitted using a quadratic and a linear function. The point of intersection is approximately located at $n=40 \mathrm{~K}$. For one million particles, the FMM takes about 1.5 seconds. To obtain these computation times, we magnetized cubes of different sizes.

computation of magnetic forces in every step (instead of every tenth) and hence might lead to more accurate magnetic forces.

\subsection{Magnetization}

Simulating the magnetization of the ferrofluid is equivalent to solving the linear Eq. (16) which is trivial in a conjugate gradient framework in which matrix-vector multiplications $G x$ are implemented. Here, $\boldsymbol{x}$ is any vector of length $3 N$ with $N$ being the number of particles.

In the conjugate gradient method for least squares, we need to evaluate the gradient using the adjoint operator $G^{\top}$. Fortunately, $G$ is symmetric which can easily be seen from Eq. $(12,13)$. There are several properties which can be exploited when solving this least squares problem. Eq. (12) reduces to a dipole magnetic field expression if $r$ is large and $W(r, h)$ becomes zero. This makes it possible to use the FMM [Beatson and Greengard 1997] to accelerate the summation in Eq. (13) for particles which are far away. During the solving process, the particles' positions are fixed, so the tree structures of the FMM does not need to be reconstructed in every iteration, saving the expensive overhead. We use the FMMTL library [Cecka and Layton 2015] to calculate the summation. The performance comparison is illustrated in Figure 7.

Since we use a linear magnetization model, $\Gamma$ is constant, and Eq. (16) is a pure linear equation. A least squares solution is guaranteed as it is convex. We can use the analytical step size for the update which only requires one evaluation of the expensive $G$ operator saving the effort of a line search. The solution $\boldsymbol{b}$ at a previous time step is used as a warm start.

Within the FMMTL library [Cecka and Layton 2015], the solver for the electric potential of a cluster of point charges is already implemented. It is is based on the same equation as magnetic and gravitational potentials. One part of it has to be modified in order to use a cluster of dipole charges as sources.

This part is called the source-to-multipole transfer [Beatson and Greengard 1997; Cecka and Layton 2015]. For $k$ point charges, this 
transfer has the following structure (Eq. (5.16) in Beatson and Greengard [1997]):

$$
{ }_{p} M_{n}^{m}=\int \sum_{s=1}^{k} \rho^{n} Y_{n}^{-m}(\alpha, \beta) q_{s} \delta\left(\boldsymbol{r}-\boldsymbol{r}_{s}\right) \mathrm{d} \boldsymbol{r},
$$

where $(\rho, \alpha, \beta)$ is the source location represented in spherical coordinates, $p M_{n}^{m}$ is the multipole expansion for point charges of degree $n$, order $m$. The charge amount for source $s$ is denoted by $q_{s}$ and the spherical harmonics function of degree $n$, order $-m$ is denoted by $Y_{n}^{-m}$. The exponentiation of $\rho$ to the power of $n$ is denoted by $\rho^{n}$.

Dipole charges have a right hand side term $\nabla \cdot \boldsymbol{m}_{s} \delta\left(\boldsymbol{r}-\boldsymbol{r}_{s}\right)$ in Poisson's Eq. (11) different from the point charges $-q_{s} \delta\left(\boldsymbol{r}-\boldsymbol{r}_{s}\right)$. We modify this part in Eq. (27). After integration by parts, we obtain

$$
{ }_{d} M_{n}^{m}=\sum_{s=1}^{k} \nabla\left(\rho_{s}^{n} Y_{n}^{-m}\left(\alpha_{s}, \beta_{s}\right)\right) \cdot \boldsymbol{m}_{s},
$$

where ${ }_{d} M_{n}^{m}$ is the multipole expansion coefficient for dipole charges and $\boldsymbol{m}_{s}$ is the magnetic dipole moment for particle $s$. After this simple modification, the FMM program for electric charges can be easily used for the evaluation of $G$.

We formulate the external field in Eq. (14) also as the one generated from magnetic particles: the same $G$ instance is used, but external particles are treated as sources. External evaluations only have to be performed once as the external field is unchanged throughout the magnetization solving process.

\subsection{Force Evaluation}

For the evaluation of the force tensor in the far field, we also use the FMM to further accelerate the computations. The force tensor is exactly the negative Hessian of a potential function $\phi$ in Cartesian coordinates:

$$
T=\mu_{0} \nabla H=-\mu_{0} \nabla(\nabla \phi) .
$$

Since our far-field potential function is evaluated in spherical coordinates, cumbersome derivations are needed in order to obtain the force tensor in Cartesian coordinates. The details of these derivations can be found in the supplemental material (S2).

\section{NUMERICAL EXAMPLES}

In this section, we present several numerical examples to study the capabilities of our approach and illustrate the influence of the different components of our model. We further demonstrate that we are able to qualitatively reproduce the behavior of real ferrofluids. In this regard, our simulations are validated against theoretical predictions and real experiments. A summary of the computation times of the different examples is provided in Table 1 and an overview of the parameters employed throughout this section can be found in Table 2.

\subsection{Fluid Crown}

In the absence of an external magnetic field, our approach is reduced to a SPH fluid simulation with surface tension. In order to validate that this fluid simulation part is working appropriately, we simulated the canonical example of an additional droplet falling into the fluid. The round shapes on the tip of the fluid and the typical shape of
Table 1. This table provides an overview of the computation time needed for simulating different numerical examples. We do not list the bunny and helix showering examples from Section 5.7 and 5.9 because the number of particles is varying in these cases. However, the example of the ferrofluid climbing up the helix presented in Section 5.10 provides an estimation for the number of particles and computation time for the bunny and helix showering examples. Moreover, the test cases illustrating the relevance of the different components of our model require a similar number of particles and consume the same computation times as their control cases, so we are also not listing each test case in this table. In all of these simulations, we make use of the FMM. All simulation times are measured on a desktop computer with two Intel ${ }^{\circledR} \mathrm{E} 5-2687 \mathrm{w}$ v4 CPUs each clocked at $3.0 \mathrm{GHz}$ and 256 GB DDR-RAM. The SPH part runs on one NVIDIA ${ }^{\circledR}$ GeForce ${ }^{\circledR}$ GTX 1080, and the FMM uses two GTX 1080 GPUs and 24 CPU cores for high performance computations.

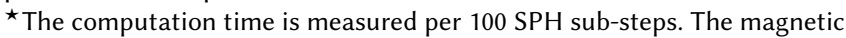
force is calculated every $10 \mathrm{SPH}$ sub-steps, taking about $70 \%$ of the total runtime. A scene usually takes around $[400,1200] \times 100$ fluid sub-steps.

\begin{tabular}{llll}
\hline Section & Scene Description & Particles & Runtime $^{\star}$ \\
\hline 5.1 & Fluid Crown & $482 \mathrm{~K}$ & $16.6 \mathrm{~s}$ \\
5.2 & Varying Surface Tension and Field Strength & $48 \mathrm{~K}$ & $11.7 \mathrm{~s}$ \\
5.5 & Dipole Experiments & $98 \mathrm{~K}$ & $25.3 \mathrm{~s}$ \\
5.6 & Field Line Orientation & $60 \mathrm{~K}$ & $15.1 \mathrm{~s}$ \\
5.8 & Ferrofluid Attracted by a Steel Sphere & $320 \mathrm{~K}$ & $90.0 \mathrm{~s}$ \\
5.10 & Ferrofluid Climbing Up a Helix & $230 \mathrm{~K}$ & $62.5 \mathrm{~s}$ \\
\hline
\end{tabular}
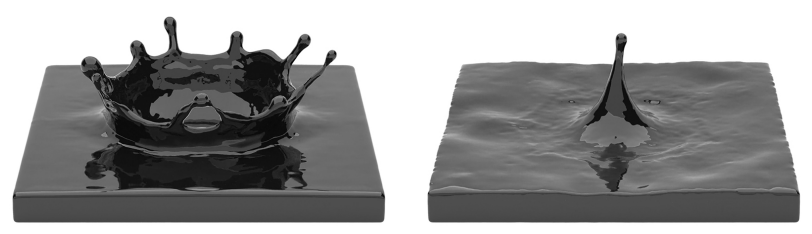

Fig. 8. Illustration of the fluid crown example in the absence of an external magnetic field. The round shapes on the tip of the fluid and the typical shape of the fluid crown can be observed here as a consequence of surface tension.

the fluid crown can be observed here as a consequence of surface tension. This is illustrated in Figure 8.

\subsection{Varying Surface Tension and Field Strength}

When being exposed to a (vertical) external magnetic field, ferrofluids form their characteristic spikes. This phenomenon is called the normal-field instability [Rosensweig 1988]. Figure 2 shows the influence of surface tension and the magnetic field strength on the shape of the spikes: a strong surface tension force smooths the contour, while a strong field strength increases the height of the spikes. Nine experiments are conducted in which the surface tension coefficients (pair-wise force coefficients) vary from $0.2 \cdot 10^{8}$ over $0.5 \cdot 10^{8}$ to $1.0 \cdot 10^{8} \mathrm{~N} / \mathrm{kg}^{2}$ and the field strengths from $1.1 \cdot 10^{4}$ over $1.2 \cdot 10^{4}$ to $1.3 \cdot 10^{4} \mathrm{~A} / \mathrm{m}$.

In order to allow for a more quantitative comparison against specific data obtained in real experiments, we systematically increase the strength of the vertical magnetic field while measuring the heights of the resulting spikes within the ferrofluid domain. This is 
Table 2. This table provides an overview of the (parameter) setup for the simulations. The size of the particles is listed in meters chosen in a way that they appropriately capture the fine details of the spikes. We observe typical dimensions of the spikes within $[3,10] \mathrm{mm}$. We choose the same size parameter $h$ for the magnetic kernel, and set the maximum number of iterations to 20. The susceptibility is taken from Gollwitzer et al. [2007] except for the dipole experiments in which we adjust the parameters based on the height of the spikes. For describing the strength of surface tension, the pair-wise force coefficients are listed according to Yang et al. [2017]. Coefficients in the range of $10^{8} \mathrm{~N} / \mathrm{kg}^{2}$ are used chosen in a way that surface tension and magnetic forces share the same magnitude. Sound speed, viscosity and artificial viscosity parameters are used according to the description by Adami et al. [2012]. Relatively high sound speeds are used in order to keep the density invariant leading to a stiff system that evolves slowly. Viscosity and artificial viscosity damp the simulation and increase numerical stability in turbulent cases. However, it shows no crucial impact on the spike shapes. The strength of the external magnetic field is chosen in such a way that it allows for the accurate formation of the spikes. The time step sizes are listed in seconds.

\begin{tabular}{|c|c|c|c|c|c|c|c|c|c|}
\hline Section & Scene Description & Particle Size $(h / \mathrm{m})$ & Susceptibility $(\chi)$ & Surface Tension & Sound Speed & Viscosity & Artificial Viscosity & External Object & Time Stepize $(\Delta t / \mathrm{s})$ \\
\hline 5.1 & Fluid Crown & $5.0 \cdot 10^{-4}$ & 1.2 & $1.9 \cdot 10^{8}$ & 9.0 & $1.0 \cdot 10^{-3}$ & 0.40 & No Magnet & $3.0 \cdot 10^{-5}$ \\
\hline 5.2 & Surface Tension and Field Strength & $5.0 \cdot 10^{-4}$ & 1.2 & {$[0.2,1.0] \cdot 10^{8}$} & 9.0 & $1.0 \cdot 10^{-3}$ & 0.04 & Vertically Constant & $3.0 \cdot 10^{-5}$ \\
\hline 5.5 & Dipole Experiments & $2.5 \cdot 10^{-4}$ & 0.8 & $16.0 \cdot 10^{8}$ & 9.0 & $1.0 \cdot 10^{-3}$ & 0.04 & Cylindrical Magnet & $3.3 \cdot 10^{-5}$ \\
\hline 5.6 & Field Line Orientation & $5.0 \cdot 10^{-4}$ & 1.2 & $0.4 \cdot 10^{8}$ & 16.0 & $1 \cdot 10^{-3}$ & 0.04 & Rotating Cuboid Magnet & $3.3 \cdot 10^{-5}$ \\
\hline 5.8 & Ferrofluid Attracted by a Steel Sphere & $5.0 \cdot 10^{-4}$ & 1.2 & $1.5 \cdot 10^{8}$ & 9.0 & $5 \cdot 10^{-3}$ & 0.40 & Magnetized Sphere & $3.0 \cdot 10^{-5}$ \\
\hline 5.7 & Ferrofluid Interacting with the Bunny & $5.0 \cdot 10^{-4}$ & 1.2 & $2.0 \cdot 10^{8}$ & 10.0 & $5 \cdot 10^{-3}$ & 0.40 & Bunny-shaped Magnet & $3.0 \cdot 10^{-5}$ \\
\hline $5.9-5.10$ & Ferrofluid Interacting with the Helix & $5.0 \cdot 10^{-4}$ & 1.2 & $2.0 \cdot 10^{8}$ & 10.0 & $2 \cdot 10^{-3}$ & 0.40 & Helix-shaped Magnet & $3.0 \cdot 10^{-5}$ \\
\hline
\end{tabular}

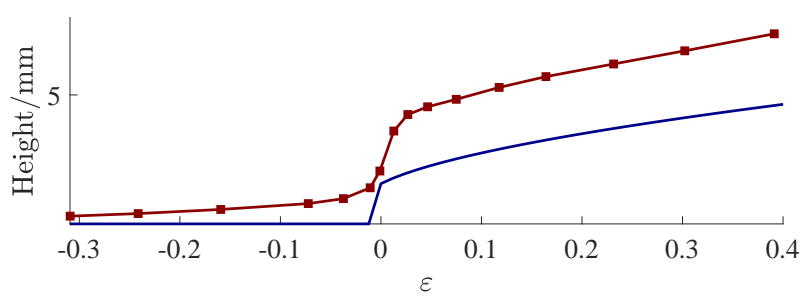

Fig. 9. Illustration of the dependence of the average height of the formed spikes on the bifurcation parameter $\varepsilon$. Our simulation (red squares) successfully reproduces the qualitative trend shown in real data (blue curve; [Gollwitzer et al. 2007], Figure 12) while the height of the spikes differs due to different surface tension strengths. The formation of the spikes can precisely be observed around $\varepsilon=0$ in the simulation and in reality.

illustrated in Figure 9 showing a comparison of the results obtained by our simulations with experimental data taken from Gollwitzer et al. [2007] (see Figure 12 therein). The spikes are formed once a critical field strength of $B=B_{c}$ is reached which corresponds to a bifurcation parameter $\varepsilon=\left(B^{2}-B_{c}^{2}\right) / B_{c}^{2}=0$. The critical strength $B_{c}$ is fitted from the measured curve. As it can clearly be observed, the simulations carried out with our approach can successfully reproduce the qualitative trend shown in real experimental data. The spike formation rapidly takes place after $\varepsilon=0$ in the simulation as in the real experiment. However, the height of the spikes in the simulation is higher than in the real experiment. This is caused by the use of a linear magnetization model neglecting the saturation of the magnetization in strong fields leading to a highly magnetized fluid. If the field strength is below the critical one, our simulation shows small elevations (not spikes). The magnetic force deforms the SPH surface but is not strong enough to form spikes.

\subsection{Levitation Artifacts}

The surface force term of the Kelvin force model points outward of the ferrofluid. It moves the surface particles away from the fluid body and causes fluid volume expansion. We resolve this artifacts by keeping negative pressures which penalizes fluid volume expansion. Figure 10a shows a snapshot before the spikes are formed. On the left, we do not penalize fluid expansion, and the surface particles float away from the bulk liquid. This case uses the high field strength and low surface tension setup from Section 5.2.

\subsection{Comparison of Center and Fitted Forces}

The center (see Eq. (19)) and fitted force (see Eq. (26)) models produce generally equivalent shape structures in homogeneous fields as illustrated in Figure 10b. However, the dynamical sequence shows more fluctuation in the case of the center force model. This case uses the medium field strength and medium surface tension from Section 5.2 except different force models.

\subsection{Experiments using a Dipole Source}

In this example, we are lifting a tray of ferrofluid located above a magnetic dipole source. Initially, the ferrofluid is packed in a square cube on the center of the tray with a radius of $2.5 \mathrm{~cm}$. It is then released freely under the influence of gravity and the external magnetic field causes the formation of the spikes almost instantly. The tray is then lifted and as a consequence the spikes are flattened as illustrated in Figure 11. We make use of sufficiently small particles $(h=0.25 \mathrm{~mm})$ in order to achieve a high resolution. We choose the surface tension coefficient so that the ferrofluid in the absence of a magnetic field has the same thickness like water drops on a plastic plane. We then set up a proper susceptibility and field strength to control the size of the spikes and make sure that the spikes disappear when the tray is lifted about $2 \mathrm{~cm}$ as shown in the real experiment. The magnet is a cylinder with a radius of $2 \mathrm{~cm}$ and a height of $8 \mathrm{~cm}$ with constant $M=140 \mathrm{kA} / \mathrm{m}$. It is represented by thousands of magnetic particles occupying the space of the cylinder. The top of the cylinder is initially located $2 \mathrm{~cm}$ below the tray. We tune the parameters to match the magnitude, density, width of spikes in the web-lab experiment. We find the fluid parameters to be quite close to the ones of real ferrofluids, and the magnetization of external magnets close to the ones of real magnets.

5.5.1 Comparison of Center versus Fitted Forces. We again compare the center and the fitted force models. In contrast to the comparison in Section 5.4, we are now making use of the dipole setup described above. As illustrated in Figure 12a, the center force formulation leads to unstable results when employing the SPH fluid solver used in this 

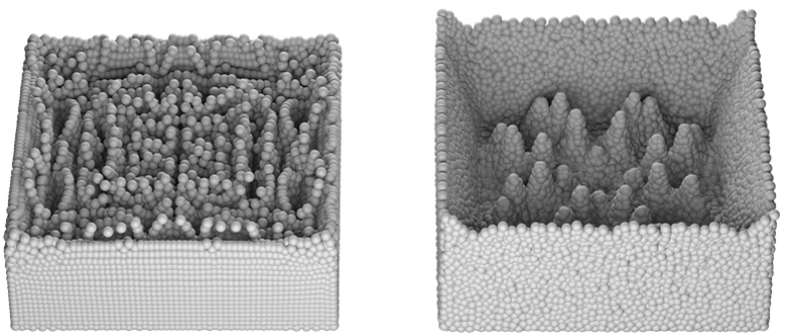

(a) The Kelvin force model has an outward surface force term, causing surface levitation artifacts and volume expansion. On the left, we only enforce volume compression. On the right, the artifacts are resolved if we value both fluid compression and expansion.
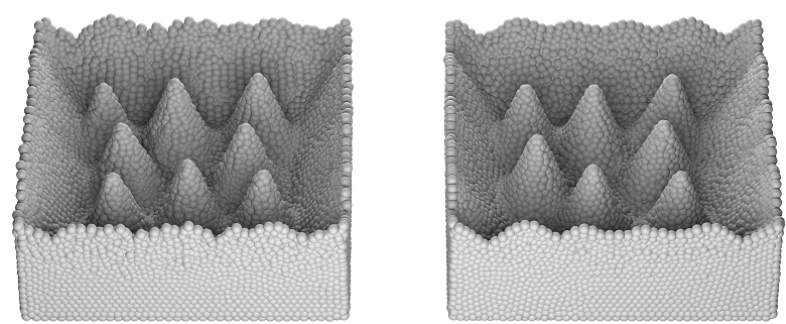

(b) Center (left) and fitted (right) forces models produce identical shape structures in homogeneous fields. However, the dynamical comparison shows the presence of more fluctuations when only the center forces are present.

Fig. 10. Illustration of the relevance to appropriately handle levitation artifacts (a), and a comparison of the center and fitted force models (b). In these simulations, constant magnetic fields are employed.
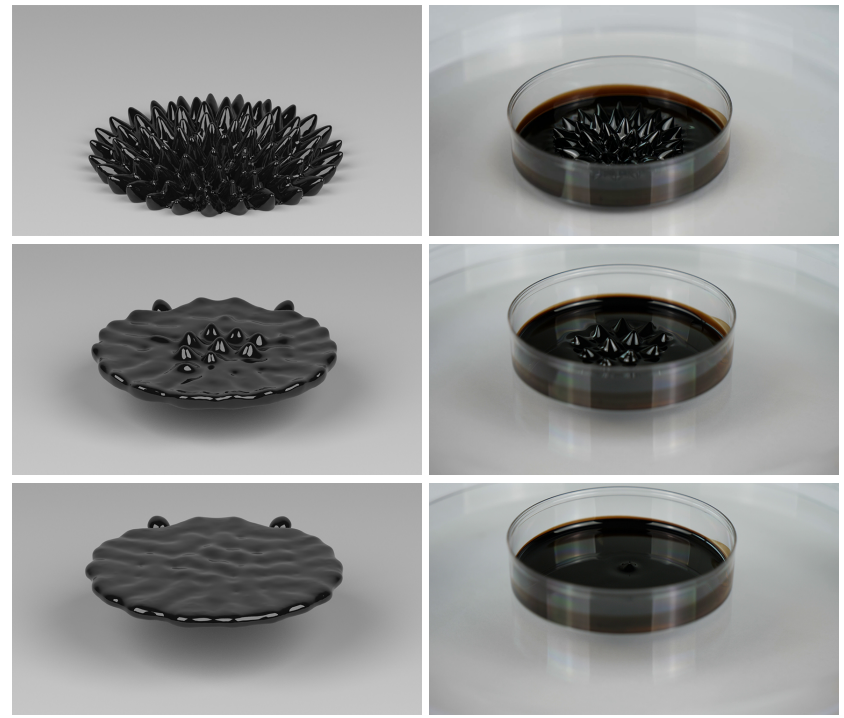

Fig. 11. Illustration of our lifting experiments of a tray of ferrofluid above a dipole source. The results of our simulation (left) and the real experiment (right) are shown.

paper within the magnetic field setup. This shows the importance of our fitted force model.

5.5.2 Relevance of Inter-particle Magnetic Forces. Modeling interparticle magnetic forces is inherently relevant in order to allow for the formation of the characteristic spikes. As illustrated in Figure $12 \mathrm{~b}$, the spikes cannot be generated if inter-particle magnetic forces are not included.

5.5.3 Varying Numbers of Iterations. The evaluation of the magnetic forces requires usually at least around 10 iterations in order to solve the magnetic field accurately. Reducing the number of iterations would make the simulation less accurate, but more efficient. Interestingly, even when solving the magnetic field with only one iteration, we get visually similar results as illustrated in Figure 12c. In more dynamical scenes, this is different.

5.5.4 Varying Kernel Sizes. In our simulation, the magnetic kernel (cubic; support of $2 h$ ) and the SPH kernel (quintic spline kernel; support of $3 h$ ) share the same parameter $h$. However, it is possible to choose a larger kernel parameter $h_{\text {mag }}$ for the magnetic part. A too-small magnetic kernel leads to strong magnetic forces when two particles are close, introducing instabilities. For evaluation purposes, we tested four different parameter setups: $h_{\text {mag }}=(0.7,1.0,1.3,1.6) h_{\mathrm{SPH}}$. While $0.7 h_{\mathrm{SPH}}$ lead to instability, the other three setups are barely distinguishable as illustrated in Figure $12 \mathrm{~d}$. A slightly smoother contour is observed for larger kernels.

\subsection{Field Line Orientation}

We rotate the magnet as shown in Figure 13 to illustrate the importance of the orientation of the field lines in order to form the characteristic spikes. Our simulation reproduces the expected phenomenon observed in real experiments.

\subsection{Magnetization of Complex Geometries}

In this section, we address the magnetization of complex geometries. We constructed a virtual magnet which has the shape of the wellknown Stanford bunny [Levoy et al. 2005]; see Figure 15. It is essentially a "frozen" ferrofluid which we coated with non-magnetizable liquid particles. What appears in the rendering as a black bunny is actually the coated layer which contains of five layers of particles. These layers prevent direct contact between the ferrofluid particles and the strong magnetic particles potentially causing instability. Beneath the coating layer, the actual "frozen" ferrofluid particles are located.

In particular, we constructed the bunny-shaped magnet as follows. First, a cubic volume is filled with ferrofluid particles before all particles outside the bunny geometry (within the cubic volume) are removed. We set the susceptibility of these particles to a sufficiently high value $(\chi=5000)$ in order to mimic the ferromagnetic properties of the material. A cylinder magnet with constant $M=1000 \mathrm{~A} / \mathrm{m}$ is placed below the bunny in order to magnetize it. We apply the 

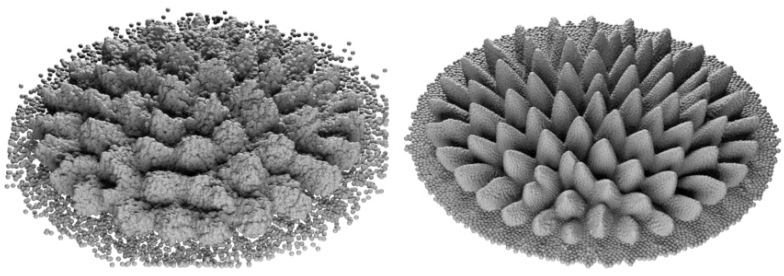

(a) When employing a dipole field, the importance of our fitted force model is even more evident compared to the case of the constant magnetic field shown before in Figure 10b: the center force model (left) leads to unstable results compared to the fitted force model (right).
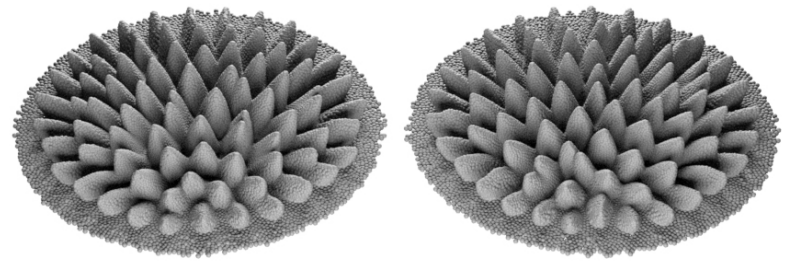

(c) Using only a single iteration for solving the magnetic field leads to qualitative equivalent results (left) compared to 20 iterations (right).
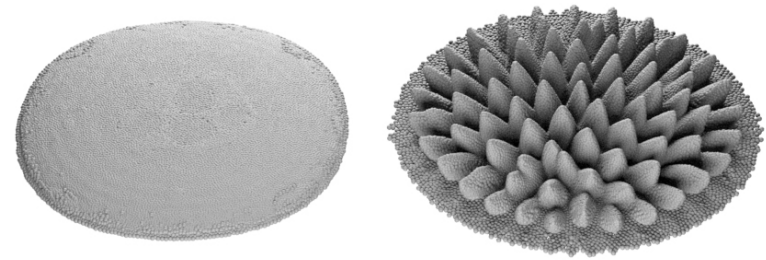

(b) Comparison of the simulation with disabled (left) and enabled (right) inter-particle forces. As it can clearly be observed, modeling inter-particle magnetic forces is inherently relevant for the formation of the characteristic spikes.

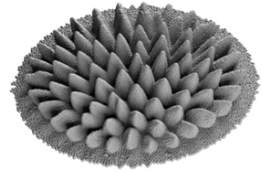

1.0

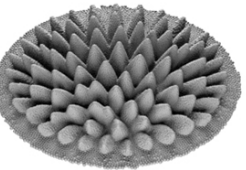

1.3

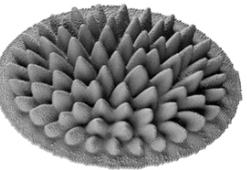

1.6 (d) We compare different kernel sizes. As it can clearly be observed, the different visual results are barely distinguishable.

Fig. 12. Illustration of different experiments using a tray of ferrofluid located above a magnetic dipole source.
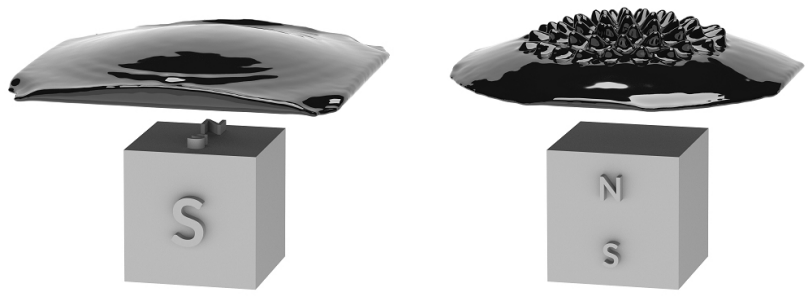

Fig. 13. If the magnetic field lines are oriented almost in parallel to the ferrofluid's surface, no spikes can be observed (left). An appropriate rotation of the magnet causing a proper reorientation of the field lines leads to the presence of spikes (right).

magnetization solver to the bunny-shaped ferrofluid so that each particle of the bunny-shaped magnet has its own orientation and strength. Such strength and orientation are fixed henceforward. Finally, we can multiply the strength of these particles by a certain number in order to control the strength of bunny-shaped magnet in the simulation. Using this procedure, we can produce magnets with almost arbitrary shapes by simply magnetizing and "freezing" the ferrofluid. However, when the coating layer is not thick enough or the field is too strong, some ferrofluid would penetrate the coating layer and after a while the whole simulation would become unstable. Improving the boundary handling techniques may solve this penetration problem.

\subsection{Ferrofluid Attracted by a Steel Sphere}

In this example, a steel sphere is magnetized by a cylindrical magnet located below. The strength of the magnet is changed in time, resulting in varying shapes of the attracted ferrofluid as shown in Figure 14.

\subsection{Ferrofluid Flowing Down a Helix}

In order to allow for a qualitative comparison with more complex geometry, we set up a simulation in which we pour a ferrofluid down the top of a steel helix placed above a strong electromagnet. We manufactured a helix of identical shape and perform a wet lab experiment with real ferrofluid. The results are shown in Figure 16. Our simulation leads to a visually comparable result providing a qualitative benchmark. Please note that we do not explicitly calibrate our simulation parameters with respect to the precise dimensions of the spikes observed in the real experiment.

\subsection{Ferrofluid Climbing Up a Helix}

Inspired by the artwork of Kodama [2008], we make use of the steel helix setup from Section 5.9 and surround the helix's lower part with ferrofluid as shown in Figure 17. As it can be seen, with increasing electric current, the ferrofluid is attracted to the top of the helix. This can successfully be reproduced using our simulation approach; see also Figure 1. Please note that in reality, the ferrofluid advances along the edges of the helix because the gradient of the magnetic field strength is higher there. Due to the coating layer, our actual magnetized helix is about $3 \mathrm{~mm}$ away from the ferrofluid. Hence, the tip of the coating layer is not the most attractive part to the ferrofluid. This explains that in the simulation the spikes are advancing along the broad band of the helix rather than along the edges. 

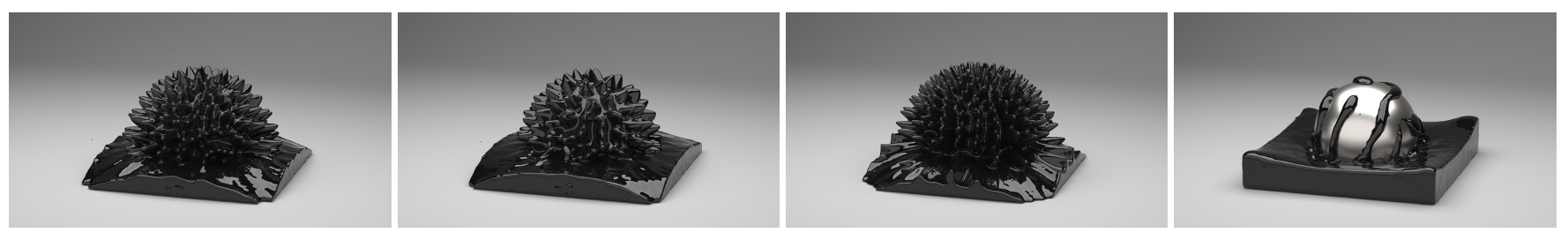

Fig. 14. In this example, a steel sphere is magnetized by a cylindrically-shaped magnet located below. The sphere attracts the ferrofluid. During the simulation, the strength of the magnet is changed over time resulting in varying fluid shapes (from left to right: medium, low, high, zero strength).
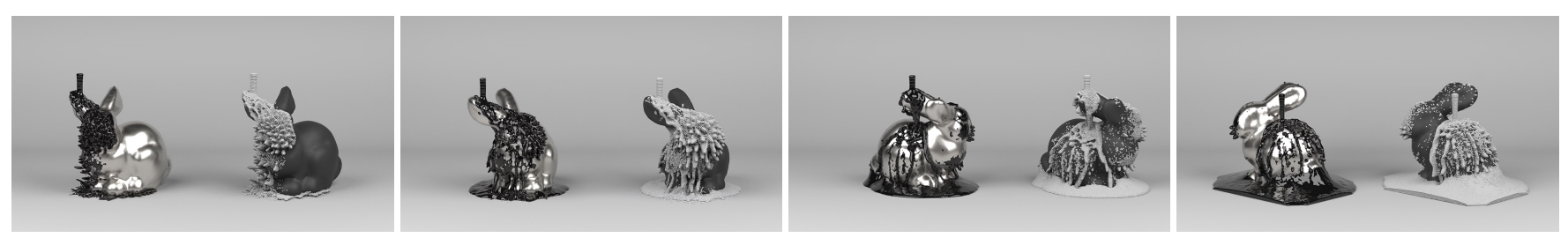

Fig. 15. We are able to simulate magnets of arbitrary shapes. In this example, the geometry of the well-known Stanford bunny is used. The magnetic bunny is showered with ferrofluid which is forming its characteristic spikes and patterns on the surface. The final rendering is shown on the left side of each frame while a particle view is presented on the right side.
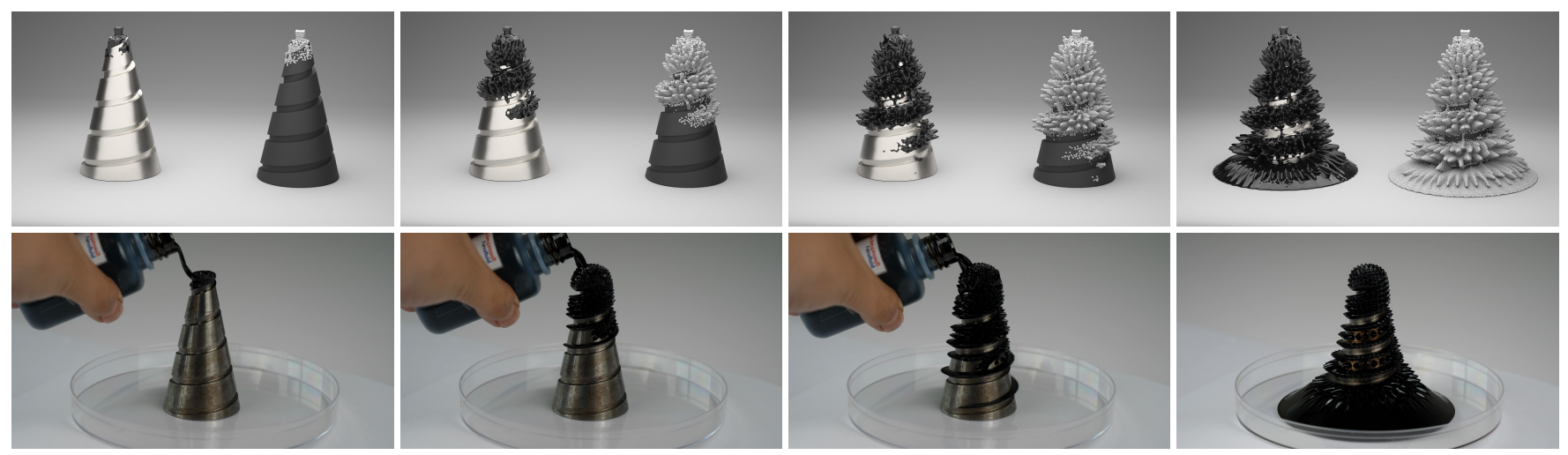

Fig. 16. In order to allow for a qualitative comparison with complex geometry, we perform a real experiment (bottom row) in which we pour a ferrofluid down the top of a steel helix placed above a strong electromagnet. Our simulation (top row; left: final rendering, right: particle view) leads to a visually comparable result.
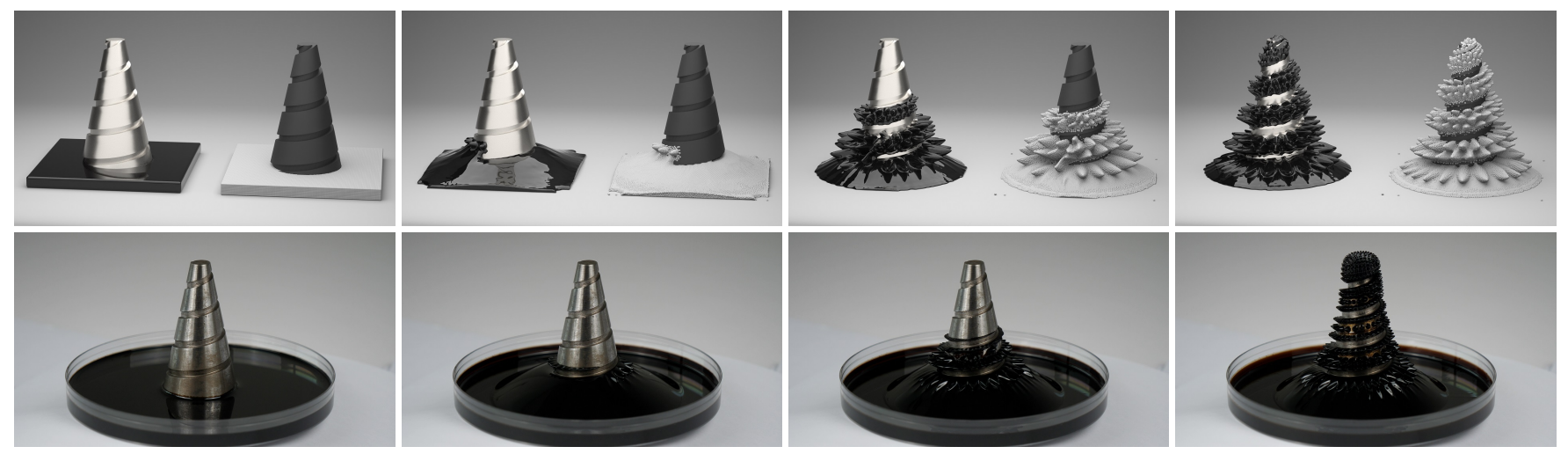

Fig. 17. We make use of the same steel helix as shown in Figure 16 which is magnetized in order to attract ferrofluid from its base to its top. The ferrofluid is literally climbing up the helix as the field strength increases as shown in this real experiment (bottom row). The simulation (top row; left: final rendering, right: particle view) can reproduce this observation as presented here. 


\section{DISCUSSION}

As illustrated in Figure 11 and 16-17, we are able to accurately reproduce key defining properties of the dynamics of ferrofluids including the formation of the characteristic spikes at the surface.

We consider the smooth magnets concept as our core contribution. We use this concept to derive the analytical magnetic field without singularities if the magnetization field is discretized as a cluster of smooth magnets. The fitted force model as an accurate numerical integration of the Kelvin force between particles is derived by precomputing the integration partially. Both the approximate central force and the accurate fitted force are bounded even when two particles perfectly overlap. Such a bounded force model ensures the stability in a particle simulation system where particles can easily get too close. The smooth magnets reduce to point magnets in the far field. Hence, this approach is immediately suitable for the FMM. It can clearly be observed that our approach scales linearly compared to the naive approach of quadratic complexity. This allows for high accuracy and efficiency as demonstrated in Section 5.

Since the dynamical behavior of ferrofluids often seems to be quite complex, our approach allowing for their efficient and large-scale numerical simulation can potentially open the door for a deeper understanding of such materials and the identification of new application cases within and beyond the field of visual computing. We consider the presented simulations as easily reproducible for the scientific community since our approach can be conveniently incorporated into a framework implementing a FMM and a SPH fluid solver covering surface tension. We further ensure reproducible by disclosing all relevant details of our implementation and provide all physical and numerical parameters in Table 2.

\subsection{Limitations and Future Work}

In this contribution, we employ a linear magnetization law Eq. (1). As part of future work activities, we can incorporate a more accurate nonlinear magnetization law into our approach. With the help of FMM, the complexity of the simulation becomes linear, but it is still computationally expensive.

The Kelvin force model lead to an outward surface force term, which forces us to limit fluid expansion to resolve the levitation artifacts. Switching to the current loop model with an inward magnetic surface force term may solve this artifacts. However the interparticle force violates momentum conservation when two particles overlap.

We believe the boundary handling and pressure solving schemes of the SPH framework can significantly be improved. However, a detailed evaluation in this regard is not done yet and we consider the integration of additional fluid solvers in order to potentially increase the degree of efficiency as an option for future work. Moreover, the application of fluid solvers covering additional aspects such as multiphase flow would increase the possibilities of our framework.

We also noticed that our magnetic solver is essentially a novel direct Poisson solver using only Lagrangian particles. It projects a field $\boldsymbol{M}$ to a divergence free field $\boldsymbol{M}+\boldsymbol{H}$ where $\nabla \cdot \boldsymbol{H}=-\nabla \cdot \boldsymbol{M}$. However, the limitation of this Poisson solver is currently its restriction to the handling of vanishing boundary condition at infinity if it is used to project the velocity field into a divergence-free field. Without a proper handling of the non-penetrating boundary condition $\boldsymbol{v} \cdot \boldsymbol{n}=0$, the fluid will penetrate the boundary. We thought about adding ghost particles at the outside of the boundary borrowing an idea from electrostatics that induced charges emerging at the boundary. This is possible future work, but at the moment it remains to be solved how to determine the induced charge particles. Please note, that all incompressible SPH variants solve a Poisson equation on Lagrangian particles. Most of them discretize the Laplacian [Bender and Koschier 2017; Ihmsen et al. 2014a; Solenthaler and Pajarola 2009] using the kernel function and solve a linear system. Our Poisson solver does not discretize the Laplacian, but instead integrate the right hand side with fundamental solutions to the Poisson equation. In this regard, this Poisson solver is similar to the strategy employed by He et al. [2012] where integral approaches are used.

\section{ACKNOWLEDGMENTS}

This work has been supported by KAUST under Individual Baseline Funding. The authors are grateful to Jan Scheffczyk and Jens Schneider for technical support. The helpful discussions with Wolfgang Heidrich, Franziska Lissel, Dmitry A. Lyakhov, Sören Pirk, Jing Ren, Ravi Samtaney, and Han Shao as well as the valuable comments of the anonymous reviewers are gratefully acknowledged.

\section{REFERENCES}

Stefan Adami, Xiangyu Hu, and Nikolaus A. Adams. 2012. A generalized wall boundary condition for smoothed particle hydrodynamics. F. Comput. Phys. 231, 21 (2012), 7057-7075.

Nadir Akinci, Gizem Akinci, and Matthias Teschner. 2013. Versatile surface tension and adhesion for SPH fluids. ACM Trans. Graph. 32, 6, Article 182 (Nov. 2013), 8 pages.

Nadir Akinci, Markus Ihmsen, Gizem Akinci, Barbara Solenthaler, and Matthias Teschner. 2012. Versatile Rigid-fluid Coupling for Incompressible SPH. ACM Trans. Graph. 31, 4, Article 62 (July 2012), 8 pages.

Matteo Antuono, Andrea Colagrossi, and Salvatore Marrone. 2012. Numerical diffusive terms in weakly-compressible SPH schemes. Computer Physics Communications 183 (Dec. 2012), 2570-2580.

Stefan Band, Christoph Gissler, Markus Ihmsen, Jens Cornelis, Andreas Peer, and Matthias Teschner. 2018a. Pressure boundaries for implicit incompressible SPH. ACM Transactions on Graphics (TOG) 37, 2 (2018), 14.

Stefan Band, Christoph Gissler, Andreas Peer, and Matthias Teschner. 2018b. MLS pressure boundaries for divergence-free and viscous SPH fluids. Computers \& Graphics 76 (2018), 37-46.

Rick Beatson and Leslie Greengard. 1997. A short course on fast multipole methods. In Wavelets, Multilevel Methods and Elliptic PDEs. Oxford University Press, 1-37.

Ted Belytschko, Yury Krongauz, D. Organ, Mark Fleming, and Petr Krysl. 1996. Meshless methods: An overview and recent developments. Computer Methods in Applied Mechanics and Engineering 139, 1 (1996), 3 - 47.

Jan Bender and Dan Koschier. 2017. Divergence-free SPH for incompressible and viscous fluids. IEEE Transactions on Visualization and Computer Graphics 23, 3 (March 2017), 1193-1206.

Denis Brousseau, Ermanno F. Borra, and Simon Thibault. 2007. Wavefront correction with a 37-actuator ferrofluid deformable mirror. Opt. Express 15, 26 (Dec 2007), 18190-18199.

James V. Byrne. 1977. Ferrofluid hydrostatics according to classical and recent theories of the stresses. Proceedings of the Institution of Electrical Engineers 124, 11 (November 1977), 1089-1097.

Yuan Cao and Ze J. Ding. 2014. Formation of hexagonal pattern of ferrofluid in magnetic field. fournal of Magnetism and Magnetic Materials 355 (2014), 93-99.

Cris Cecka and Simon Layton. 2015. FMMTL: FMM Template Library a generalized framework for kernel matrices. In Numerical Mathematics and Advanced Applications, ENUMATH 2013, Assyr Abdulle, Simone Deparis, Daniel Kressner, Fabio Nobile, and Marco Picasso (Eds.). Springer International Publishing, Cham, 611-620.

Andreas Engel. 2001. Comment on "Invalidation of the Kelvin Force in Ferrofluids" Phys. Rev. Lett. 86 (May 2001), 4978-4978. Issue 21.

Chuyuan Fu, Qi Guo, Theodore Gast, Chenfanfu Jiang, and Joseph Teran. 2017. A polynomial particle-in-cell method. ACM Trans. Graph. 36, 6, Article 222 (Nov. 2017), 
12 pages.

Christian Gollwitzer, Gunar Matthies, Reinhard Richter, Ingo Rehberg, and Lutz Tobiska 2007. The surface topography of a magnetic fluid: a quantitative comparison between experiment and numerical simulation. Journal of Fluid Mechanics 571 (2007), 455474.

Herb Hartshorne, Christopher J Backhouse, and William E Lee. 2004. Ferrofluid-based microchip pump and valve. Sensors and Actuators B: Chemical 99, 2 (2004), 592 600 .

Xiaowei He, Ning Liu, Sheng Li, Hongan Wang, and Guoping Wang. 2012. Local Poisson SPH For Viscous Incompressible Fluids. Comput. Graph. Forum 31, 6 (Sept. 2012), 1948-1958.

Xiaowei He, Huamin Wang, Fengjun Zhang, Hongan Wang, Guoping Wang, and Kun Zhou. 2014. Robust simulation of sparsely sampled thin features in SPH-based free surface flows. ACM Trans. Graph. 34, 1, Article 7 (Dec. 2014), 9 pages.

Yuanming Hu, Yu Fang, Ziheng Ge, Ziyin Qu, Yixin Zhu, Andre Pradhana, and Chenfanfu Jiang. 2018. A moving least squares material point method with displacement discontinuity and two-way rigid body coupling. ACM Trans. Graph. 37, 4, Article 150 (July 2018), 14 pages.

Markus Ihmsen, Nadir Akinci, Markus Becker, and Matthias Teschner. 2011. A Parallel SPH Implementation on Multi-Core CPUs. Computer Graphics Forum 30, 1 (2011) 99-112.

Markus Ihmsen, Jens Cornelis, Barbara Solenthaler, Christopher Horvath, and Matthias Teschner. 2014a. Implicit incompressible SPH. IEEE Transactions on Visualization and Computer Graphics 20, 3 (March 2014), 426-435.

Markus Ihmsen, Jens Orthmann, Barbara Solenthaler, Andreas Kolb, and Matthias Teschner. 2014b. SPH Fluids in Computer Graphics. In Eurographics 2014 - State of the Art Reports, Sylvain Lefebvre and Michela Spagnuolo (Eds.). The Eurographics Association.

Tomokazu Ishikawa, Yonghao Yue, Kei Iwasaki, Yoshinori Dobashi, and Tomoyuki Nishita. 2012. Visual simulation of magnetic fluid taking into account dynamic deformation in spikes. In Image Electronics and Visual Computing Workshop.

Tomokazu Ishikawa, Yonghao Yue, Kei Iwasaki, Yoshinori Dobashi, and Tomoyuki Nishita. 2013. Visual simulation of magnetic fluid using a procedural approach for spikes shape. In Computer Vision, Imaging and Computer Graphics. Theory and Application, Gabriela Csurka, Martin Kraus, Robert S. Laramee, Paul Richard, and José Braz (Eds.). Springer Berlin Heidelberg, Berlin, Heidelberg, 112-126.

Chenfanfu Jiang, Craig Schroeder, and Joseph Teran. 2017. An angular momentum conserving affine-particle-in-cell method. f. Comput. Phys. 338, C (June 2017) 137-164.

Seung-Wook Kim, Sun Young Park, and Junghyun Han. 2018. Magnetization dynamics for magnetic object interactions. ACM Trans. Graph. 37, 4, Article 121 (July 2018), 13 pages.

Sachiko Kodama. 2008. Dynamic ferrofluid sculpture: organic shape-changing art forms Commun. ACM 51, 6 (June 2008), 79-81.

Dan Koschier, Jan Bender, Barbara Solenthaler, and Matthias Teschner. 2019. EUROGRAPHICS Tutorial on Smoothed Particle Hydrodynamics Techniques for the Physics Based Simulation of Fluids and Solids. (2019).

Olga Lavrova, Gunar Matthies, Teodora Mitkova, Viktor Polevikov, and Lutz Tobiska. 2006. Numerical treatment of free surface problems in ferrohydrodynamics. Fournal of Physics: Condensed Matter 18, 38 (2006), S2657.

Olga Lavrova, Gunar Matthies, and Lutz Tobiska. 2008. Numerical study of solitonlike surface configurations on a magnetic fluid layer in the Rosensweig instability. Communications in Nonlinear Science and Numerical Simulation 13, 7 (2008), 1302310.

Marc Levoy, John Gerth, Brian Curless, and KariPulli. 2005. The Stanford 3D scanning repository. (2005).

Steven Lind, Rui Xu, Peter K. Stansby, and Benedict D. Rogers. 2012. Incompressible Smoothed Particle Hydrodynamics for Free-surface Flows: A Generalised Diffusionbased Algorithm for Stability and Validations for Impulsive Flows and Propagating Waves. F. Comput. Phys. 231, 4 (Feb. 2012), 1499-1523.

Mario Liu. 2001. Liu replies. Phys. Rev. Lett. 86 (May 2001), 4979-4979. Issue 21.

Kenta Mitsufuji, Shuhei Matsuzawa, Katsuhiro Hirata, and Fumikazu Miyasaka. 2016. Meshless method employing magnetic moment method and particle method for magnetic fluid motion analysis. IEEf Journal of Industry Applications 5, 4 (2016), $355-359$.

Masafumi Miwa, H. Harita, T. Nishigami, R. Kaneko, and H. Unozawa. 2003. Frequency characteristics of stiffness and damping effect of a ferrofluid bearing. Tribology Letters 15, 2 (01 Aug 2003), 97-105.

Joseph J. Monaghan. 1992. Smoothed particle hydrodynamics. Annual review of astronomy and astrophysics 30 (1992), 543-574

Joseph J. Monaghan. 2012. Smoothed Particle Hydrodynamics and Its Diverse Applications. Annual Review of Fluid Mechanics 44, 1 (2012), 323-346.

Matthias Müller, David Charypar, and Markus Gross. 2003. Particle-based fluid simulation for interactive applications. In Proceedings of the 2003 ACM SIGGRAPH/Eurographics Symposium on Computer Animation (SCA '03). Eurographics Association, Aire-la-Ville, Switzerland, Switzerland, 154-159.
Ricardo H. Nochetto, Abner J. Salgado, and Ignacio Tomas. 2016a. A diffuse interface model for two-phase ferrofluid flows. Computer Methods in Applied Mechanics and Engineering 309 (2016), 497-531.

Ricardo H. Nochetto, Abner J. Salgado, and Ignacio Tomas. 2016b. The equations of ferrohydrodynamics: modeling and numerical methods. Mathematical Models and Methods in Applied Sciences 26, 13 (2016), 2393-2449.

Stefan Odenbach. 2008. Ferrofluids: Magnetically Controllable Fluids and Their Applications. Vol. 594. Springer.

Stefan Odenbach and Mario Liu. 2001. Invalidation of the kelvin force in ferrofluids Phys. Rev. Lett. 86 (Jan 2001), 328-331. Issue 2.

Andreas Peer, Markus Ihmsen, Jens Cornelis, and Matthias Teschner. 2015. An implicit viscosity formulation for SPH fluids. ACM Transactions on Graphics (TOG) 34,4 (2015), 114.

Andreas Peer and Matthias Teschner. 2017. Prescribed velocity gradients for highly viscous SPH fluids with vorticity diffusion. IEEE transactions on visualization and computer graphics 23, 12 (2017), 2656-2662.

Kuldip Raj, B. Moskowitz, and R. Casciari. 1995. Advances in ferrofluid technology. fournal of Magnetism and Magnetic Materials 149, 1 (1995), 174-180. Proceedings of the Seventh International Conference on Magnetic Fluids.

Ronald E. Rosensweig. 1987. Magnetic Fluids. 19 (01 1987), 437-463.

Ronald E. Rosensweig. 1988. An Introduction To Ferrohydrodynamics. Chemical Engineering Communications 67, 1 (1988), 1-18.

Ronald E. Rosensweig. 1997. Ferrohydrodynamics. Dover Publications.

Barbara Solenthaler and Renato Pajarola. 2009. Predictive-corrective incompressible SPH. ACM Trans. Graph. 28, 3, Article 40 (July 2009), 6 pages.

Pengnan Sun, Andrea Colagrossi, Salvatore Marrone, and A. M. Zhang. 2017. The $\delta$ plus-SPH model: Simple procedures for a further improvement of the SPH scheme. Computer Methods in Applied Mechanics and Engineering 315 (March 2017), 25-49.

Alexandre Tartakovsky and Paul Meakin. 2005. Modeling of surface tension and contact angles with smoothed particle hydrodynamics. Phys. Rev. E 72 (Aug 2005), 026301. Issue 2 .

Bernhard Thomaszewski, Andreas Gumann, Simon Pabst, and Wolfgang Straßer. 2008 Magnets in motion. ACM Trans. Graph. 27, 5, Article 162 (Dec. 2008), 9 pages.

Kalarickaparambil J. Vinoy and Rakesh M. Jha. 1996. Radar absorbing materials: from theory to design and characterization. Boston : Kluwer Academic Publishers.

Tao Yang, Ralph R. Martin, Ming C. Lin, Jian Chang, and Shi-Min Hu. 2017. Pairwise force SPH model for real-time multi-interaction applications. IEEE Transactions on Visualization and Computer Graphics 23, 10 (Oct 2017), 2235-2247.

Gaku Yoshikawa, Katsuhiro Hirata, Fumikazu Miyasaka, and Yu Okaue. 2010. Numerical analysis of transitional behavior of ferrofluid employing MPS method and FEM. In Digests of the 2010 14th Biennial IEEE Conference on Electromagnetic Field Computation. 1-1.

Markus Zahn. 2001. Magnetic fluid and nanoparticle applications to nanotechnology. fournal of Nanoparticle Research 3, 1 (Feb 2001), 73-78.

Wen Zheng, Bo Zhu, Byungmoon Kim, and Ronald Fedkiw. 2015. A new incompressibility discretization for a hybrid particle MAC grid representation with surface tension. 7. Comput. Phys. 280, C (Jan. 2015), 96-142.

Yongning Zhu and Robert Bridson. 2005. Animating sand as a fluid. ACM Trans. Graph. 24, 3 (July 2005), 965-972.

\section{APPENDIX}

Table 3. Polynomial coefficients of $C_{1}: q \mapsto C_{1}(q)$.

\begin{tabular}{rrrrr}
\hline & $0 \leq q<1$ & $1 \leq q<2$ & $2 \leq q<3$ & \multicolumn{1}{c}{$3 \leq q<4$} \\
\hline$a_{4}$ & $9.978 \cdot 10^{-9}$ & $-2.764 \cdot 10^{-9}$ & $-1.096 \cdot 10^{-9}$ & $3.799 \cdot 10^{-10}$ \\
$a_{3}$ & $-2.979 \cdot 10^{-8}$ & $2.869 \cdot 10^{-8}$ & $9.770 \cdot 10^{-9}$ & $-6.263 \cdot 10^{-9}$ \\
$a_{2}$ & $2.389 \cdot 10^{-9}$ & $-9.945 \cdot 10^{-8}$ & $-2.547 \cdot 10^{-8}$ & $3.947 \cdot 10^{-8}$ \\
$a_{1}$ & $4.531 \cdot 10^{-8}$ & $1.251 \cdot 10^{-7}$ & $2.650 \cdot 10^{-9}$ & $-1.135 \cdot 10^{-7}$ \\
$a_{0}$ & $2.446 \cdot 10^{-11}$ & $-2.370 \cdot 10^{-8}$ & $5.007 \cdot 10^{-8}$ & $1.274 \cdot 10^{-7}$ \\
\hline
\end{tabular}

Table 4. Polynomial coefficients of $C_{2}: q \mapsto C_{2}(q)$.

\begin{tabular}{rrrrr}
\hline & $0 \leq q<1$ & $1 \leq q<2$ & $2 \leq q<3$ & \multicolumn{1}{c}{$3 \leq q<4$} \\
\hline$a_{4}$ & $6.695 \cdot 10^{-8}$ & $-3.084 \cdot 10^{-8}$ & $3.504 \cdot 10^{-9}$ & $7.334 \cdot 10^{-10}$ \\
$a_{3}$ & $-1.617 \cdot 10^{-7}$ & $2.291 \cdot 10^{-7}$ & $-5.259 \cdot 10^{-8}$ & $-9.588 \cdot 10^{-9}$ \\
$a_{2}$ & $1.682 \cdot 10^{-8}$ & $-5.883 \cdot 10^{-7}$ & $2.788 \cdot 10^{-7}$ & $4.370 \cdot 10^{-8}$ \\
$a_{1}$ & $1.345 \cdot 10^{-7}$ & $5.611 \cdot 10^{-7}$ & $-6.241 \cdot 10^{-7}$ & $-7.480 \cdot 10^{-8}$ \\
$a_{0}$ & $1.109 \cdot 10^{-10}$ & $-1.144 \cdot 10^{-7}$ & $4.918 \cdot 10^{-7}$ & $2.341 \cdot 10^{-8}$ \\
\hline
\end{tabular}

